\title{
The temporal evolution of neutral modes in the impulsively started flow through a circular pipe and their connection to the nonlinear stability of Hagen-Poiseuille flow
}

\author{
By ANDREW G. WALTON \\ Mathematics Department, Imperial College of Science, Technology and Medicine, \\ 180 Queen's Gate, London SW7 2BZ, UK
}

(Received 2 January 2001 and in revised form 23 October 2001)

The linear stability of the impulsively started flow through a pipe of circular crosssection is studied at high Reynolds number $R$. A crucial non-dimensional time of $O\left(R^{7 / 9}\right)$ is identified at which the disturbance acquires internal flow characteristics. It is shown that even if the disturbance amplitude at this time is as small as $O\left(R^{-22 / 27}\right)$ the subsequent evolution of the perturbation is nonlinear, although it can still be followed analytically using a multiple-scales approach. The amplitude and wave speed of the nonlinear disturbance are calculated as functions of time and we show that as $t \rightarrow \infty$, the disturbance evolves into the long-wave limit of the neutral mode structure found by Smith \& Bodonyi in the fully developed Hagen-Poiseuille flow, into which our basic flow ultimately evolves. It is proposed that the critical amplitude found here forms a stability boundary between the decay of linear disturbances and 'bypass' transition, in which the fully developed state is never attained.

\section{Introduction}

In the middle of the nineteenth century an exact solution to the Navier-Stokes equations was found for the steady flow of a fluid through a straight pipe of circular cross-section. The solution has become known as Hagen-Poiseuille flow (HPF) in honour of the two researchers who independently discovered the experimental law relating the axial pressure gradient along the pipe to the mean velocity of the flow. If a carefully controlled experiment is performed it is indeed possible to realize HPF: however in many practical situations the disturbance environment is such that this solution only exists over a finite range of Reynolds number or in some situations not at all. Attention therefore focused on the study of the stability of this flow, with the first approach incorporating the effects of viscosity due to Sexl (1927), who found the flow to be linearly stable to axisymmetric disturbances at high Reynolds number. Since this early work there have been many theoretical investigations (e.g. Gill 1965, 1973; Davey \& Drazin 1969), and numerical studies (Salwen \& Grosch 1972; Garg \& Rouleau 1972). For a more complete list the reader is referred to the paper by Draad, Kuiken \& Nieuwstadt (1998). The general conclusion of these studies is that HPF is stable to all linear disturbances and that the least-damped modes at high Reynolds number have azimuthal wavenumbers $N=0$ (axisymmetric) and $N=1$. This latter mode is also found to give the largest amplification through transient growth (Schmid \& Henningson 1994). 
Ever since the fundamental experiments of Reynolds (1883) it has been thought that nonlinearity must play a particularly important role in the transition process for this flow, and full numerical simulations (e.g. O'Sullivan \& Breuer 1994) have reproduced qualitatively some of the key phenomena observed in experiments, such as the generation of puffs and slugs of vorticity (Wygnanski \& Champagne 1973). A review of the many experimental studies that have been performed can be found in a recent paper by Han, Tumin \& Wygnanski (2000).

As far as theoretical approaches are concerned the key nonlinear study has been that of Smith \& Bodonyi (1982a) (hereinafter referred to as SB). They found the existence of a nonlinear neutral wave structure at asymptotically large Reynolds number with a disturbance amplitude of $O\left(R^{-1 / 3}\right)$ and wavenumber and frequency of $O(1)$, and showed that in the limit of small wavenumber $\alpha$, the disturbance amplitude is reduced to $O\left(R^{-1 / 3} \alpha^{5 / 3}\right)$. SB studied the nonlinear equilibrium critical layer that arises in the flow field and as a result of a numerical investigation proposed that this structure only admits the $N=1$ neutral mode. This has recently been proved rigorously by Walton (2001). The significance of the $N=1$ mode in a number of independent studies suggests that it is of particular significance for the pipe flow transition problem. We will see this again in the work we present later in this paper.

There have been a number of attempts to find the SB neutral modes numerically at finite values of the Reynolds number by a continuation process involving for example, the slow rotation of the pipe (Toplosky \& Akylas 1998; Barnes \& Kerswell 2000) but so far this approach has been unsuccessful. At present it is not known with any certainty whether the SB modes are isolated or whether they are connected in some way to the linear stability of the developing flow in the pipe. It is this final issue that provides the motivation for the work presented in this paper. We consider the basic flow to be the impulsively started unsteady motion in the pipe (which is an exact solution of the Navier-Stokes equations and possesses HPF as its large-time limit) and begin by determining its linear stability structure at high Reynolds number. We then show that the upper branch modes can be rendered nonlinear by increasing their amplitude to a threshold value. It is then possible for a fixed wavelength disturbance to remain neutral beyond the upper branch time provided it adjusts its amplitude and frequency in a specific way over the time scale associated with the mean flow. This idea has formed the basis of a number of recent nonlinear stability theories including vortex-wave interaction (Hall \& Smith 1991) and the PSE approach (Herbert 1997). In the present context the nonlinear temporal evolution of the neutral modes can be determined analytically and we find that the $N=1$ mode matches exactly to the SB structure mentioned earlier as $t \rightarrow \infty$.

The structure of the paper is as follows. In the remainder of this section we introduce the governing equations and the basic flow under study. In $\S 2$ we derive the upper branch linear stability properties of this flow at high Reynolds number. Section 3 concerns the details of the linear critical layer and how the phase shift induced across it is affected by increasing the disturbance size slightly. The effects of nonlinearity and its implications for the various scalings are discussed and set the scene for the investigation of $\S 4$ where the temporal evolution of the neutral state is considered in the nonlinear regime. Although most of the analysis can be tackled analytically, the temporal evolution of the nonlinear disturbance amplitude and wave speed need to be determined numerically and this is performed in $\S 5$. In $\S 6$ we demonstrate how our nonlinear structure matches back exactly to the linear upper branch structure as $t \rightarrow 0$ and, more significantly perhaps, how it matches to the SB modes in HPF as $t \rightarrow \infty$. Finally, in $\S 7$ we draw some conclusions and suggest avenues for further study. 


\subsection{The governing equations and basic flow}

The cylindrical polar coordinate system $(a x, a r, \theta)$ with origin at the pipe entrance is used throughout this paper, where $a x$, $a r$ and $\theta$ represent the axial, radial and azimuthal coordinates respectively. The $(x, r, \theta)$ velocity components are written as $\left(g a^{2} / \rho v\right)(u, v, w)$, where $4 g$ is the constant axial pressure gradient to be applied to the pipe. Here, $a$ is the radius of the pipe and the constants $\rho$ and $v$ are the density and kinematic viscosity of the incompressible fluid. We express the pressure as $\left(g^{2} a^{4} / \rho v^{2}\right) p$, and the time is written in the form $(\rho v / g a) t$. These scalings enable us to write the governing three-dimensional, unsteady Navier-Stokes equations in the non-dimensional form:

$$
\begin{gathered}
\frac{\partial u}{\partial x}+\frac{\partial v}{\partial r}+\frac{v}{r}+\frac{1}{r} \frac{\partial w}{\partial \theta}=0 \\
\frac{\partial u}{\partial t}+u \frac{\partial u}{\partial x}+v \frac{\partial u}{\partial r}+\frac{w}{r} \frac{\partial u}{\partial \theta}=-\frac{\partial p}{\partial x}+\frac{1}{R}\left(\frac{\partial^{2} u}{\partial x^{2}}+\frac{\partial^{2} u}{\partial r^{2}}+\frac{1}{r} \frac{\partial u}{\partial r}+\frac{1}{r^{2}} \frac{\partial^{2} u}{\partial \theta^{2}}\right) \\
\frac{\partial v}{\partial t}+u \frac{\partial v}{\partial x}+v \frac{\partial v}{\partial r}+\frac{w}{r} \frac{\partial v}{\partial \theta}-\frac{w^{2}}{r} \\
=-\frac{\partial p}{\partial r}+\frac{1}{R}\left(\frac{\partial^{2} v}{\partial x^{2}}+\frac{\partial^{2} v}{\partial r^{2}}+\frac{1}{r} \frac{\partial v}{\partial r}+\frac{1}{r^{2}} \frac{\partial^{2} v}{\partial \theta^{2}}-\frac{v}{r^{2}}-\frac{2}{r^{2}} \frac{\partial w}{\partial \theta}\right), \\
\frac{\partial w}{\partial t}+u \frac{\partial w}{\partial x}+v \frac{\partial w}{\partial r}+\frac{w}{r} \frac{\partial w}{\partial \theta}+\frac{v w}{r} \\
=-\frac{1}{r} \frac{\partial p}{\partial \theta}+\frac{1}{R}\left(\frac{\partial^{2} w}{\partial x^{2}}+\frac{\partial^{2} w}{\partial r^{2}}+\frac{1}{r} \frac{\partial w}{\partial r}+\frac{1}{r^{2}} \frac{\partial^{2} w}{\partial \theta^{2}}-\frac{w}{r^{2}}+\frac{2}{r^{2}} \frac{\partial v}{\partial \theta}\right),
\end{gathered}
$$

where the Reynolds number $R$ is defined by

$$
R=\frac{g a^{3}}{\rho v^{2}} .
$$

We consider the basic flow to be parallel and unsteady, with

$$
u=u_{0}(r, t), \quad v=w=0,
$$

and in terms of the non-dimensional variables, the pressure gradient

$$
\frac{\partial p}{\partial x}=-\frac{4}{R}
$$

is suddenly applied to the pipe at time $t=0$ say, thus setting the fluid into motion. From substitution into (1), the governing equation for $u_{0}$ is

$$
\frac{\partial u_{0}}{\partial t}=\frac{1}{R}\left(4+\frac{\partial^{2} u_{0}}{\partial r^{2}}+\frac{1}{r} \frac{\partial u_{0}}{\partial r}\right),
$$

and the initial and boundary conditions are

$$
\begin{gathered}
u_{0}=0 \quad \text { at } t=0 \text { for } 0 \leqslant r \leqslant 1, \quad u_{0}=0 \text { at } r=1 \text { for } t \geqslant 0, \\
u_{0} \text { finite at } r=0 .
\end{gathered}
$$


The exact solution has the well-known form (e.g. Szymanski 1932; Batchelor 1967):

$$
u_{0}(r, t)=1-r^{2}-8 \sum_{n=1}^{\infty} \frac{J_{0}\left(j_{o n} r\right)}{j_{o n}^{3} J_{1}\left(j_{o n}\right)} \exp \left(-j_{o n}^{2} t / R\right),
$$

where $j_{o n}$ is the $n$th zero of the Bessel function $J_{0}$. This solution will be valid for all $t \leqslant O(R)$, provided we are considering the flow at a distance of $O(R)$ downstream of the pipe entrance. For values of time such that $t \ll O(R)$, it can easily be shown that to leading order the solution acquires the two-tiered form

$$
u_{0}(r, t)= \begin{cases}4 t / R, & r \sim O(1) \\ (4 t / R) u_{B}(\eta), & r=1-(t / R)^{1 / 2} \eta,\end{cases}
$$

where

$$
u_{B}(\eta)=\left(1+\frac{1}{2} \eta^{2}\right) \operatorname{erf}\left(\frac{1}{2} \eta\right)-\frac{1}{2} \eta^{2}+\frac{\eta}{\sqrt{\pi}} \exp \left(-\eta^{2} / 4\right) .
$$

The analytic form (3) of the basic flow is an attractive one to study because as $t \rightarrow 0$ it displays external flow characteristics and is similar to a Blasius boundarylayer flow (as can be seen from the two-tiered form (4)), while as $t \rightarrow \infty, u_{0} \rightarrow 1-r^{2}$, i.e. HPF, whose stability characteristics, as discussed earlier, still remain something of a mystery. Our aim is to shed light on the nonlinear stability of HPF by considering it as the long-time limit of the impulsively started problem described above.

\section{The linear stability of the impulsively started flow}

For the purposes of the linear stability analysis of this section we will consider the basic flow in its two-tiered form (4). Later, when we consider the nonlinear regime in $\S 4$, it will be necessary to use expression (3) for $u_{0}$.

We begin by imposing a small three-dimensional disturbance upon the basic flow. We write

$$
(u, v, w, p)=\left(u_{0}, 0,0, p_{0}-4 R^{-1} x\right)+\delta(\hat{u}, \hat{v}, \hat{w}, \hat{p}),
$$

where $p_{0}$ is a constant and $\delta$ is a small quantity representing the amplitude of the disturbance. For the present, $\delta$ can be regarded as arbitrary but later in $\S 3$ we will suggest a critical value for it which then allows the disturbance to subsequently evolve in a nonlinear fashion as the basic flow becomes more developed. In this section we wish to study how the linear neutral stability criteria alter as the base flow evolves in time. Substitution into the Navier-Stokes equations (1) leads to the following set of linear equations:

$$
\begin{gathered}
\frac{\partial \hat{u}}{\partial x}+\frac{\partial \hat{v}}{\partial r}+\frac{\hat{v}}{r}+\frac{1}{r} \frac{\partial \hat{w}}{\partial \theta}=0 \\
\frac{\partial \hat{u}}{\partial t}+u_{0} \frac{\partial \hat{u}}{\partial x}+\hat{v} \frac{\partial u_{0}}{\partial r}=-\frac{\partial \hat{p}}{\partial x}+\frac{1}{R}\left(\frac{\partial^{2} \hat{u}}{\partial x^{2}}+\frac{\partial^{2} \hat{u}}{\partial r^{2}}+\frac{1}{r} \frac{\partial \hat{u}}{\partial r}+\frac{1}{r^{2}} \frac{\partial^{2} \hat{u}}{\partial \theta^{2}}\right), \\
\frac{\partial \hat{v}}{\partial t}+u_{0} \frac{\partial \hat{v}}{\partial x}=-\frac{\partial \hat{p}}{\partial r}+\frac{1}{R}\left(\frac{\partial^{2} \hat{v}}{\partial x^{2}}+\frac{\partial^{2} \hat{v}}{\partial r^{2}}+\frac{1}{r} \frac{\partial \hat{v}}{\partial r}+\frac{1}{r^{2}} \frac{\partial^{2} \hat{v}}{\partial \theta^{2}}-\frac{\hat{v}}{r^{2}}-\frac{2}{r^{2}} \frac{\partial \hat{w}}{\partial \theta}\right), \\
\frac{\partial \hat{w}}{\partial t}+u_{0} \frac{\partial \hat{w}}{\partial x}=-\frac{1}{r} \frac{\partial \hat{p}}{\partial \theta}+\frac{1}{R}\left(\frac{\partial^{2} \hat{w}}{\partial x^{2}}+\frac{\partial^{2} \hat{w}}{\partial r^{2}}+\frac{1}{r} \frac{\partial \hat{w}}{\partial r}+\frac{1}{r^{2}} \frac{\partial^{2} \hat{w}}{\partial \theta^{2}}-\frac{\hat{w}}{r^{2}}+\frac{2}{r^{2}} \frac{\partial \hat{v}}{\partial \theta}\right),
\end{gathered}
$$

which we will refer to as the linearized Navier-Stokes (LNS) equations. These will serve as our governing equations for the remainder of this section. 
In the usual way we now express the disturbance in the wave-like form

$$
(\hat{u}, \hat{v}, \hat{w}, \hat{p})=\operatorname{Re}((F(r, t), \mathrm{i} G(r, t), H(r, t), P(r, t)) \exp (\mathrm{i} \xi)),
$$

where $\operatorname{Re}$ denotes 'the real part' and $\xi=\alpha(x-c t)+N \theta$. Throughout this paper we will be seeking neutral modes so that the streamwise wavenumber $\alpha$ and wave speed $c$ are both real with $0<c<\max \left(u_{0}\right)$. The azimuthal wavenumber $N$ must be an integer for periodicity and we will concentrate on three-dimensional modes for which $N \neq 0$.

For small values of $t$ the instability will behave as though it is in an external boundary layer: only at later times does the pipe curvature begin to play a significant role. It is at such a time that we wish to determine the disturbance structure, so to aid this we set $t=t_{0} / \varepsilon$, with $t_{0}$ of $O(1)$ and $\varepsilon$ a small parameter left undetermined for the moment. Derivatives with respect to time can then be expressed as

$$
\frac{\partial}{\partial t}=-\mathrm{i} \alpha c+\varepsilon \frac{\partial}{\partial t_{0}} .
$$

Thus, provided $\alpha c \gg O\left(\varepsilon / t_{0}\right) \gg O(1 / R), t_{0}$ can be regarded as a parameter and the flow (in the two-tiered form (4)) can be considered as quasi-steady for the purposes of deriving its linear stability structure. In view of this we will henceforth suppress the $t_{0}$ dependence and use a prime to denote differentiation with respect to the radial variable $r$. Note that the above inequalities require the Reynolds number to be asymptotically large and this restriction on the Reynolds number will be in force for the remainder of this paper. It is worth making the point that a finite Reynolds number approach, involving solving the cylindrical Orr-Sommerfeld equations, although potentially useful in determining a critical Reynolds number, would not be mathematically consistent as then there would be no distinction between the time scales over which the basic flow and wave evolve.

The solution procedure adopted in this section follows the same lines as many previous high Reynolds number analyses of parallel or nearly parallel basic flows with Smith \& Bodonyi's (1980) work on the linear stability of pipe entry flow being the closest previous work. To the best of our knowledge the linear stability of this particular basic flow has not been studied before, and we describe our analysis in some detail to facilitate the comparison and matching we will make with the nonlinear development to be outlined in $\S 4$.

\subsection{The inviscid region}

Because of the largeness of the Reynolds number, viscous effects are confined to thin layers and so across the majority of the pipe, i.e. for $O(1)$ values of $r$, the disturbance equations are, from substitution of (8) into (7),

$$
\begin{gathered}
\alpha F+G^{\prime}+\frac{G}{r}+\frac{N H}{r}=0, \quad\left(u_{0}-c\right) \alpha F+G u_{0}^{\prime}=-\alpha P, \\
\left(u_{0}-c\right) \alpha G=P^{\prime}, \quad\left(u_{0}-c\right) \alpha H=-\frac{N P}{r} .
\end{gathered}
$$

These equations can be manipulated to leave $P$ satisfying the Rayleigh equation

$$
\left(u_{0}-c\right)\left(P^{\prime \prime}+\frac{1}{r} P^{\prime}-\frac{N^{2}}{r^{2}} P-\alpha^{2} P\right)=2 u_{0}^{\prime} P^{\prime} .
$$


For convenience we define

$$
u_{0}=(4 t / R) \bar{u}_{0}, \quad c=(4 t / R) \bar{c} \quad \text { with } \quad t=t_{0} / \varepsilon
$$

so that (10) remains unchanged except that $u_{0}, c$ are replaced by $\bar{u}_{0}, \bar{c}$. Viscous effects are only important at leading order in a thin critical layer near the location where $u_{0}=c$, and near the wall where $r=1$. There are two distinguished scalings for the wave speed $c$ in terms of the Reynolds number: one has the critical layer sited within the wall layer and gives rise to the scaling on the lower branch of the linear neutral curve at high Reynolds number, while the other scaling preserves the distinction between the two layers (although $c$ is still small) and provides the upper branch behaviour. It is this latter situation which we will concentrate on here as it is found that this structure is the more relevant of the two when we move into the nonlinear regime in $\S 4$. We will return to discuss the detailed structure of the wall and critical layers later: for the moment we concentrate on the solution of the Rayleigh equation when $u_{0}$ has the two-tiered form given earlier.

In this situation the solution acquires a three-zone structure. The differences in the solution in the three regions arise essentially from the differing forms for the basic flow: in the outer zone (region I) $\bar{u}_{0}-\bar{c} \approx 1$; in region II $\bar{u}_{0}-\bar{c} \approx u_{B}(\eta)$; and in region III $\bar{u}_{0}-\bar{c} \approx \lambda_{0} \eta-\bar{c}$ (where $\bar{u}_{0} \sim \lambda_{0} \eta$ as $\eta \rightarrow 0$ ). We now discuss each zone in sufficient detail to derive the behaviour along the upper branch with the main aim of determining the Reynolds number dependence of $\alpha, c$ and $\varepsilon$.

\subsubsection{The subregions}

Region I occupies the majority of the pipe. We have $r \sim O(1)$ and $\bar{u}_{0}=1$ to leading order. The Rayleigh equation reduces to a form of Laplace's equation:

$$
P^{\prime \prime}+\frac{1}{r} P^{\prime}-\left(\frac{N^{2}}{r^{2}}+\alpha_{0}^{2}\right) P=0,
$$

where $\alpha_{0}$ is the leading-order part of the wavenumber and is assumed to be $O(1)$. The solution to this equation which remains finite as $r \rightarrow 0$ is

$$
P=\frac{I_{N}\left(\alpha_{0} r\right)}{I_{N}\left(\alpha_{0}\right)}
$$

where we have performed a normalization such that $P=1$ at $r=1$. Here, $I_{N}$ is a modified Bessel function of order $N$ : the occurrence of a term of this form provides the curvature effects referred to earlier.

In view of the form of the inner solution for $u_{0}$, region II has the radial scaling

$$
r=1-(\varepsilon R)^{-1 / 2} t_{0}^{1 / 2} \eta
$$

The basic flow and pressure expand as

$$
\bar{u}_{0}=u_{B}(\eta)+O(\varepsilon R)^{-1}, \quad P=1+(\varepsilon R)^{-1 / 2} t_{0}^{1 / 2} p_{1}(\eta)+\cdots,
$$

with $p_{1}$ subject to the matching condition

$$
p_{1} \sim-\alpha_{0} \frac{I_{N}^{\prime}\left(\alpha_{0}\right)}{I_{N}\left(\alpha_{0}\right)} \eta \quad \text { as } \quad \eta \rightarrow \infty,
$$

in view of the solution for $P$ in region I. Substitution of expansions (14), (15) into the Rayleigh equation (10) leads to the result

$$
p_{1}^{\prime}=-\alpha_{0} \frac{I_{N}^{\prime}\left(\alpha_{0}\right)}{I_{N}\left(\alpha_{0}\right)}\left(u_{B}(\eta)\right)^{2},
$$

which matches appropriately, since $u_{B}(\infty)=1$, from consideration of (5). 
In region III the wave speed $c$ makes an appearance at leading order. First, suppose the radial scaling here is $r=1-(\varepsilon R)^{-1 / 2} t_{0}^{1 / 2} q Y$, where $q$ is a small parameter to be determined in terms of $R$ and $\varepsilon$. The small- $\eta$ behaviour of $u_{B}$ can be deduced from (4), (5) and is of the form

$$
u_{B}(\eta) \sim \lambda_{0} \eta+\lambda_{1} \eta^{2} \quad \text { as } \quad \eta \rightarrow 0 \quad \text { with } \quad \lambda_{0}=\frac{2}{\sqrt{\pi}}, \lambda_{1}=-\frac{1}{2} .
$$

Thus in region III , the basic flow and the wave speed expand as

$$
\bar{u}_{0}=\lambda_{0} q Y+\lambda_{1} q^{2} Y^{2}+\cdots, \quad \bar{c}=q \bar{c}_{0}+q^{2} \bar{c}_{1}+\cdots,
$$

and the appropriate expansion for $P$ is

$$
P=1+(\varepsilon R)^{-1 / 2} t_{0}^{1 / 2} q^{3} \hat{p}_{1}+\cdots,
$$

implied by the behaviour of (16) as $\eta \rightarrow 0$. To bring the effects of the wavenumber into the solution for $\hat{p}_{1}$, we require $\partial^{2} P / \partial r^{2} \sim \alpha_{0}^{2} \sim O(1)$ and so we must have the balance $(\varepsilon R)^{1 / 2} q / t_{0}^{1 / 2} \sim O(1)$, which implies that $q \sim\left(\varepsilon R / t_{0}\right)^{-1 / 2}$, and determines the radial scaling of region III as

$$
r=1-(\varepsilon R)^{-1} t_{0} Y
$$

In terms of $\varepsilon$ and $R$, the pressure, basic flow and wave speed therefore expand as

$$
\begin{gathered}
P=1+\left(\varepsilon R / t_{0}\right)^{-2} \hat{p}_{1}(Y)+\left(\varepsilon R / t_{0}\right)^{-5 / 2} \hat{p}_{2}(Y)+\cdots, \\
\bar{u}_{0}=\left(\varepsilon R / t_{0}\right)^{-1 / 2} \lambda_{0} Y+\left(\varepsilon R / t_{0}\right)^{-1} \lambda_{1} Y^{2}+\cdots, \\
\bar{c}=\left(\varepsilon R / t_{0}\right)^{-1 / 2} \bar{c}_{0}+\left(\varepsilon R / t_{0}\right)^{-1} \bar{c}_{1}+\cdots,
\end{gathered}
$$

with a wavenumber expansion of the form

$$
\alpha=\alpha_{0}+\left(\varepsilon R / t_{0}\right)^{-1 / 2} \alpha_{1}+\cdots .
$$

Then, from substitution into the Rayleigh equation, $\hat{p}_{1}$ is controlled by

$$
\left(\lambda_{0} Y-\bar{c}_{0}\right)\left(\hat{p}_{1}^{\prime \prime}-\left(N^{2}+\alpha_{0}^{2}\right)\right)=2 \lambda_{0} \hat{p}_{1}^{\prime},
$$

with solution

$$
\hat{p}_{1}^{\prime}=D_{0}\left(\lambda_{0} Y-\bar{c}_{0}\right)^{2}-\left(N^{2}+\alpha_{0}^{2}\right)\left(Y-\bar{c}_{0} / \lambda_{0}\right) .
$$

To match to region II we require

$$
\hat{p}_{1}^{\prime} \sim-\alpha_{0} \frac{I_{N}^{\prime}\left(\alpha_{0}\right)}{I_{N}\left(\alpha_{0}\right)} \lambda_{0}^{2} Y^{2} \quad \text { as } \quad Y \rightarrow \infty,
$$

from (16), (17), thus determining the constant $D_{0}$ as

$$
D_{0}=-\alpha_{0} \frac{I_{N}^{\prime}\left(\alpha_{0}\right)}{I_{N}\left(\alpha_{0}\right)} .
$$

Since the dynamics are inviscid to this order we also require the tangential flow condition that $\hat{p}_{1}^{\prime} \rightarrow 0$ as $Y \rightarrow 0$. Application of this constraint to the solution (21) leads to the eigenrelation

$$
\alpha_{0} \bar{c}_{0} \lambda_{0} I_{N}^{\prime}\left(\alpha_{0}\right)=\left(N^{2}+\alpha_{0}^{2}\right) I_{N}\left(\alpha_{0}\right) .
$$

We will return to discuss this eigenrelation in some detail later. To find a second 
relation between $\alpha_{0}$ and $\bar{c}_{0}$ we need to proceed to the next order where we find the equation

$$
\begin{aligned}
\left(\lambda_{0} Y\right. & \left.-\bar{c}_{0}\right) \hat{p}_{2}^{\prime \prime}-2 \lambda_{0} \hat{p}_{2}^{\prime} \\
& =-\left(\lambda_{1} Y^{2}-\bar{c}_{1}\right) \hat{p}_{1}^{\prime \prime}+4 \lambda_{1} Y \hat{p}_{1}^{\prime}+\left(\lambda_{1} Y^{2}-\bar{c}_{1}\right)\left(N^{2}+\alpha_{0}^{2}\right)+2\left(\lambda_{0} Y-\bar{c}_{0}\right) \alpha_{0} \alpha_{1} .
\end{aligned}
$$

At this stage it is convenient to introduce the new radial variable

$$
\zeta=\lambda_{0} Y-\bar{c}_{0},
$$

and after substituting for $\hat{p}_{1}$, integrating, and using (22) we find that for $\zeta>0$

$$
\hat{p}_{2}^{\prime}(\zeta)=Q_{3}(\zeta)-\frac{2 \lambda_{1}\left(N^{2}+\alpha_{0}^{2}\right)}{\lambda_{0}^{4}} \zeta^{2} \ln \zeta,
$$

where $Q_{3}(\zeta)$ is a regular function of $\zeta$ (with $Q_{3} \propto \zeta$ as $\zeta \rightarrow 0$ ) that can be determined (and depends on the wavenumber correction $\alpha_{1}$ ) but is not required for our purposes. The irregular behaviour of the second term in (24) implies the existence of a viscous critical layer (which we will denote by region IV) centred around $\zeta=0$. We will leave the discussion of the dynamics of this layer until $\S 3$; at this stage we merely note the phase shift of $-\pi$ that arises there (Lin 1955; Stuart 1963; Reid 1965). The implication of this property is that the term

$$
\ln \zeta \text { for } \zeta>0 \text { is replaced by } \ln |\zeta|-\mathrm{i} \pi \text { for } \zeta<0 \text {. }
$$

In order to understand the role that the phase shift plays in the stability structure it is more convenient to work in terms of the perturbation to the normal velocity rather than the pressure. In region III, the normal velocity expands as

$$
G=\left(\frac{\varepsilon R}{t_{0}}\right)^{1 / 2} \hat{G}_{0}(\zeta)+\hat{G}_{1}(\zeta)+\cdots,
$$

where $4 \alpha_{0} \zeta \hat{G}_{0}=-\hat{p}_{1}^{\prime}(Y)$ from $(9 c)$, and therefore $\hat{G}_{0}(0)=\left(N^{2}+\alpha_{0}^{2}\right) /\left(4 \alpha_{0} \lambda_{0}\right)$. The irregular behaviour seen above in (24) arises in the normal velocity through the term $\hat{G}_{1}$, where we find

$$
\hat{G}_{1}=-\frac{\lambda_{0}}{4 \alpha_{0}} \frac{Q_{3}(\zeta)}{\zeta}+\frac{\lambda_{1}\left(N^{2}+\alpha_{0}^{2}\right)}{2 \lambda_{0}^{3} \alpha_{0}} \zeta \ln \zeta \text { for } \zeta>0,
$$

and using the phase shift property (25) of the critical layer referred to above we deduce that

$$
\hat{G}_{1}=-\frac{\lambda_{0}}{4 \alpha_{0}} \frac{Q_{3}(\zeta)}{\zeta}+\frac{\lambda_{1}\left(N^{2}+\alpha_{0}^{2}\right)}{2 \lambda_{0}^{3} \alpha_{0}} \zeta \ln (-\zeta)-\frac{\mathrm{i} \pi \lambda_{1}\left(N^{2}+\alpha_{0}^{2}\right)}{2 \lambda_{0}^{3} \alpha_{0}} \zeta \quad \text { for } \quad \zeta<0 .
$$

Thus upon approaching the pipe wall where $\zeta=-\bar{c}_{0}$ we find that, since $\hat{v}$ is the real part of $\mathrm{i} G \exp (\mathrm{i} \xi)$, and the function $Q_{3}$ is real,

$$
\begin{array}{r}
\hat{v} \rightarrow\left(-\left(\frac{\varepsilon R}{t_{0}}\right)^{3 / 2} \lambda_{0} \hat{G}_{0}^{\prime}\left(-\bar{c}_{0}\right)(1-r)-\frac{\lambda_{0}}{4 \alpha_{0}} \frac{Q_{3}\left(-\bar{c}_{0}\right)}{\bar{c}_{0}}+\frac{\lambda_{1} \bar{c}_{0}\left(N^{2}+\alpha_{0}^{2}\right)}{2 \lambda_{0}^{3} \alpha_{0}} \ln \left(\bar{c}_{0}\right)\right) \sin \xi \\
-\frac{\pi \lambda_{1} \bar{c}_{0}\left(N^{2}+\alpha_{0}^{2}\right)}{2 \lambda_{0}^{3} \alpha_{0}} \cos \xi \text { as } r \rightarrow 1,
\end{array}
$$

where $\hat{G}_{0}^{\prime}\left(-\bar{c}_{0}\right)=\left(N^{2}+\alpha_{0}^{2}\right) /\left(4 \alpha_{0} \bar{c}_{0} \lambda_{0}\right)$, from $(9 c)$ and (21). We see that the phase shift across the critical layer gives rise to a term proportional to $\cos \xi$ which must be 
matched to the behaviour in the viscous wall layer to be discussed next. The required balance of phase shifts will determine a unique scaling for $\varepsilon$ in terms of $R$. This effectively tells us how much time has elapsed from the start of the impulsive motion before the disturbances begin to acquire internal characteristics. Knowledge of $\varepsilon$ will then allow us to determine the Reynolds number dependence of the linear neutral modes.

\subsection{The wall layer}

In the wall layer (region V) we have $u_{0}-c \simeq-c \sim O\left(\varepsilon R / t_{0}\right)^{-3 / 2}$ from (11) and $(20 c)$. The balance in this layer is therefore between the disturbance frequency $\alpha c$ and the viscous operator $(1 / R) \partial^{2} / \partial r^{2}$. If we suppose that the thickness of this layer is $s$, then the aforementioned balance implies $s \sim\left(\alpha_{0} R c\right)^{-1 / 2} \sim \alpha_{0}^{-1 / 2} R^{1 / 4}\left(\varepsilon / t_{0}\right)^{3 / 4}$. Thus we introduce the wall variable $\bar{Y}$ via the scaling

$$
r=1-\alpha_{0}^{-1 / 2} R^{1 / 4}\left(\varepsilon / t_{0}\right)^{3 / 4} \bar{Y},
$$

where the order-one quantity $\alpha_{0}$ is inserted for comparison with the nonlinear structure to be outlined in $\S 4$. The basic flow here has the near-wall form

$$
u_{0}=\left(R t_{0} / \varepsilon\right)^{-1 / 4} 4 \lambda_{0} \alpha_{0}^{-1 / 2} \bar{Y}+\cdots,
$$

from (11), (17) and (27). The inviscid Rayleigh balances (9) imply that $F$ and $H$ are both $O\left(c^{-1}\right)$ in the wall layer, and so we write the perturbations to $u$ and $w$ as

$$
\begin{aligned}
\hat{u} & =\left(\varepsilon R / t_{0}\right)^{3 / 2} \alpha_{0}^{-1} \operatorname{Re}(\bar{u}(\bar{Y}) \exp (\mathrm{i} \xi))+\cdots, \\
\hat{w} & =\left(\varepsilon R / t_{0}\right)^{3 / 2} \alpha_{0}^{-1} \operatorname{Re}(\bar{w}(\bar{Y}) \exp (\mathrm{i} \xi))+\cdots .
\end{aligned}
$$

In order that the continuity equation is satisfied, the expansion for $\hat{v}$ must be

$$
\hat{v}=-\left(\varepsilon / t_{0}\right)^{9 / 4} R^{7 / 4} \alpha_{0}^{-3 / 2} \operatorname{Re}(\mathrm{i} \bar{v}(\bar{Y}) \exp (\mathrm{i} \xi))+\cdots,
$$

and we know that the wave speed has the form

$$
c=4\left(\varepsilon R / t_{0}\right)^{-3 / 2} \bar{c}_{0}+\cdots,
$$

while

$$
\hat{p}=\operatorname{Re}(\exp (\mathrm{i} \xi))+\cdots,
$$

in view of the normalization applied earlier. Substitution into the LNS equations (7) then yields the leading-order balances

$$
\alpha_{0} \bar{u}+\bar{v}^{\prime}+N \bar{w}=0, \quad-4 \mathrm{i} \bar{c}_{0} \bar{u}=-\mathrm{i} \alpha_{0}+\bar{u}^{\prime \prime}, \quad-4 \mathrm{i} \bar{c}_{0} \bar{w}=-\mathrm{i} N+\bar{w}^{\prime \prime},
$$

with these equations being subject to the matching conditions

$$
\bar{u} \rightarrow \frac{\alpha_{0}}{4 \bar{c}_{0}}, \quad \bar{w} \rightarrow \frac{N}{4 \bar{c}_{0}} \quad \text { as } \quad \bar{Y} \rightarrow \infty,
$$

implied by (9) and (11), and the no-slip wall conditions

$$
\bar{u}=\bar{v}=\bar{w}=0 \quad \text { on } \quad \bar{Y}=0 .
$$

The solution is easily found to be

$$
\begin{gathered}
\bar{u}=\frac{\alpha_{0}}{4 \bar{c}_{0}}(1-\exp (-m \bar{Y})), \quad \bar{w}=\left(N / \alpha_{0}\right) \bar{u}, \\
\bar{v}=-\frac{\left(N^{2}+\alpha_{0}^{2}\right)}{4 \bar{c}_{0}}\left(\bar{Y}-\frac{1}{m}(1-\exp (-m \bar{Y}))\right),
\end{gathered}
$$


with $m=\left(2 \bar{c}_{0}\right)^{1 / 2}(1-\mathrm{i})$. The behaviour of this solution as we move out of the wall layer is crucial for the purposes of balancing the phase shifts across the critical and wall layers as we shall see in the next section.

\subsection{The balancing of phase shifts and the large time behaviour of the} linear neutral modes

If we examine the wall layer solution (28) we find that as $\bar{Y} \rightarrow \infty$

$$
\hat{v} \sim-\left(\varepsilon / t_{0}\right)^{9 / 4} R^{7 / 4} \alpha_{0}^{-3 / 2}\left(N^{2}+\alpha_{0}^{2}\right)\left(\frac{\bar{Y}}{4 \bar{c}_{0}} \sin \xi-\frac{\sin \xi+\cos \xi}{2^{7 / 2} \bar{c}_{0}^{3 / 2}}\right) .
$$

The first term in (29) matches automatically to the first term in (26), while the second term must be of the same order of magnitude as that arising from the phase shift across the critical layer (i.e. the second term in (26)). Thus we require the order-ofmagnitude balance $\varepsilon^{9 / 4} R^{7 / 4} \sim O(1)$, giving the Reynolds number scaling for $\varepsilon$ as

$$
\varepsilon=R^{-7 / 9} \text {. }
$$

As remarked earlier this tells us that the earliest time at which internal flow characteristics are important is when $t \sim O\left(R^{7 / 9}\right)$. The terms in $\hat{v}$ proportional to $\cos \xi$ in regions III and V must match exactly, not just in magnitude, and this leads to the second eigenrelation:

$$
\frac{1}{2^{7 / 2} t_{0}^{9 / 4} \alpha_{0}^{3 / 2} \bar{c}_{0}^{3 / 2}}=-\frac{\pi \lambda_{1} \bar{c}_{0}}{2 \lambda_{0}^{3} \alpha_{0}},
$$

obtained by equating terms in (26) and (29). Eliminating $\bar{c}_{0}$ between the eigenrelations (22) and (30) we find

$$
\left(N^{2}+\alpha_{0}^{2}\right) \frac{I_{N}\left(\alpha_{0}\right)}{I_{N}^{\prime}\left(\alpha_{0}\right)}=\frac{\lambda_{0}^{11 / 5} \alpha_{0}^{4 / 5}}{2 \pi^{2 / 5}\left(-\lambda_{1}\right)^{2 / 5} t_{0}^{9 / 10}} .
$$

For given azimuthal wavenumber $N$, this equation gives the variation of the leadingorder streamwise wavenumber $\alpha_{0}$ as a function of scaled time $t_{0}$. This result, together with the corresponding lower branch eigenrelation, can be interpreted as defining a time interval over which a linear disturbance of fixed wavelength experiences growth. Relation (31) is the temporal equivalent of that obtained by Smith \& Bodonyi (1980) in their investigation of the spatial development of the linear instability in the entrance region of a circular pipe. As alluded to earlier and to be seen in more detail later, the advantage of the temporal approach adopted here is that the analytic form for our basic flow is valid for all time and allows us to connect the Blasius-like solution for small $t$ to the Hagen-Poiseuille profile obtained as $t \rightarrow \infty$. There is no direct spatial equivalent of this analytic solution because of the complications arising from non-parallel effects. The asymptotic structure of the linear neutral modes is shown schematically in figure 1 for $t_{0} \sim O(1)$, where we have used the scaling $\varepsilon=R^{-7 / 9}$ deduced above.

We can infer the scaling of the neutral modes for $O(1)$ times by letting $t_{0} \rightarrow R^{-7 / 9} t$ in (31), and we find

$$
\alpha_{0} \sim R^{7 / 12} \frac{\lambda_{0}^{11 / 6}}{2^{5 / 6}\left(-\pi \lambda_{1}\right)^{1 / 3}} t^{-3 / 4},
$$

where we have used the fact that $I_{N}\left(\alpha_{0}\right) / I_{N}^{\prime}\left(\alpha_{0}\right) \rightarrow 1$ as $\alpha_{0} \rightarrow \infty$. This result is consistent with that obtained from a full linear analysis of the stability problem at $O(1)$ times, along the lines of the work of Smith \& Bodonyi (1982b) for an accelerating boundary 


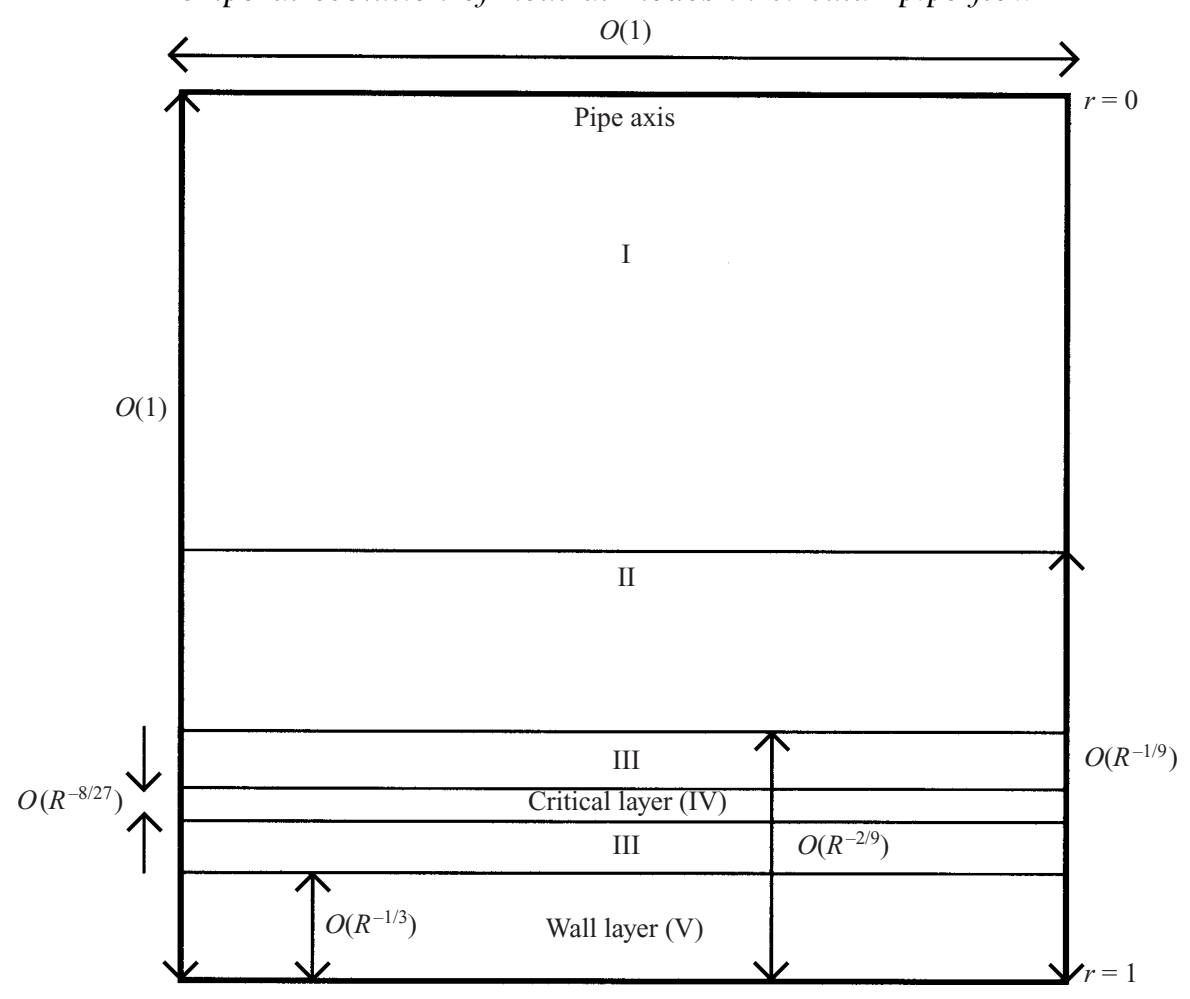

FIGURE 1. The structure of the linear neutral modes of $\S 2$ when $t \sim O\left(R^{7 / 9}\right)$.

layer. The result we obtain, namely that $\alpha_{0} \sim O\left(R^{7 / 12}\right)$, differs from theirs because of the relative smallness of our basic flow which is $O\left(R^{-1}\right)$ at $O(1)$ times.

Of greater interest to us here is how the neutral criterion alters as the basic flow becomes more developed. Letting $t_{0} \rightarrow \infty$ in (31) we obtain

$$
\alpha_{0} \sim \frac{\lambda_{0}^{11}}{32 \pi^{2} N^{5} \lambda_{1}^{2}} t_{0}^{-9 / 2}
$$

which can be re-written as $\alpha_{0} \sim 2^{8} t_{0}^{-9 / 2} /\left(\pi^{15 / 2} N^{5}\right)$, after substitution for $\lambda_{0}$ and $\lambda_{1}$ from (17). From the first eigenrelation (22) we find $\bar{c}_{0} \rightarrow N / \lambda_{0}$ as $t_{0} \rightarrow \infty$, and so

$$
c \sim\left(4 N / \lambda_{0}\right) R^{-1 / 3} t_{0}^{3 / 2} \sim 2 N \sqrt{\pi} R^{-1 / 3} t_{0}^{3 / 2},
$$

as $t_{0} \rightarrow \infty$. This linear stability structure remains valid provided $\alpha_{0} c \gg \varepsilon / t_{0} \gg 1 / R$. Substituting in for $\varepsilon, \alpha_{0}$ and $c$ from above we see that our solution therefore remains intact until

$$
t_{0} \sim O\left(R^{2 / 9}\right)
$$

i.e. until $t \sim O(R)$. At this critical time the locally neutral wave speed becomes $O(1)$, the wavenumber $O\left(R^{-1}\right)$, the two-tier structure for the basic flow merges into one and all the asymptotic regions analysed above become comparable with the pipe diameter. The thickness of the various regions is shown in figure 2 for $t=R \tilde{t}$ with $\tilde{t} \ll 1$.

Thus we obtain the result that as $t \rightarrow O(R)$, the characteristic wavelength of an upper branch neutral disturbance increases to the same length scale as the basic flow. The subsequent behaviour of the neutral modes is therefore non-parallel and it would seem that it can only be found by solving the Navier-Stokes equations 


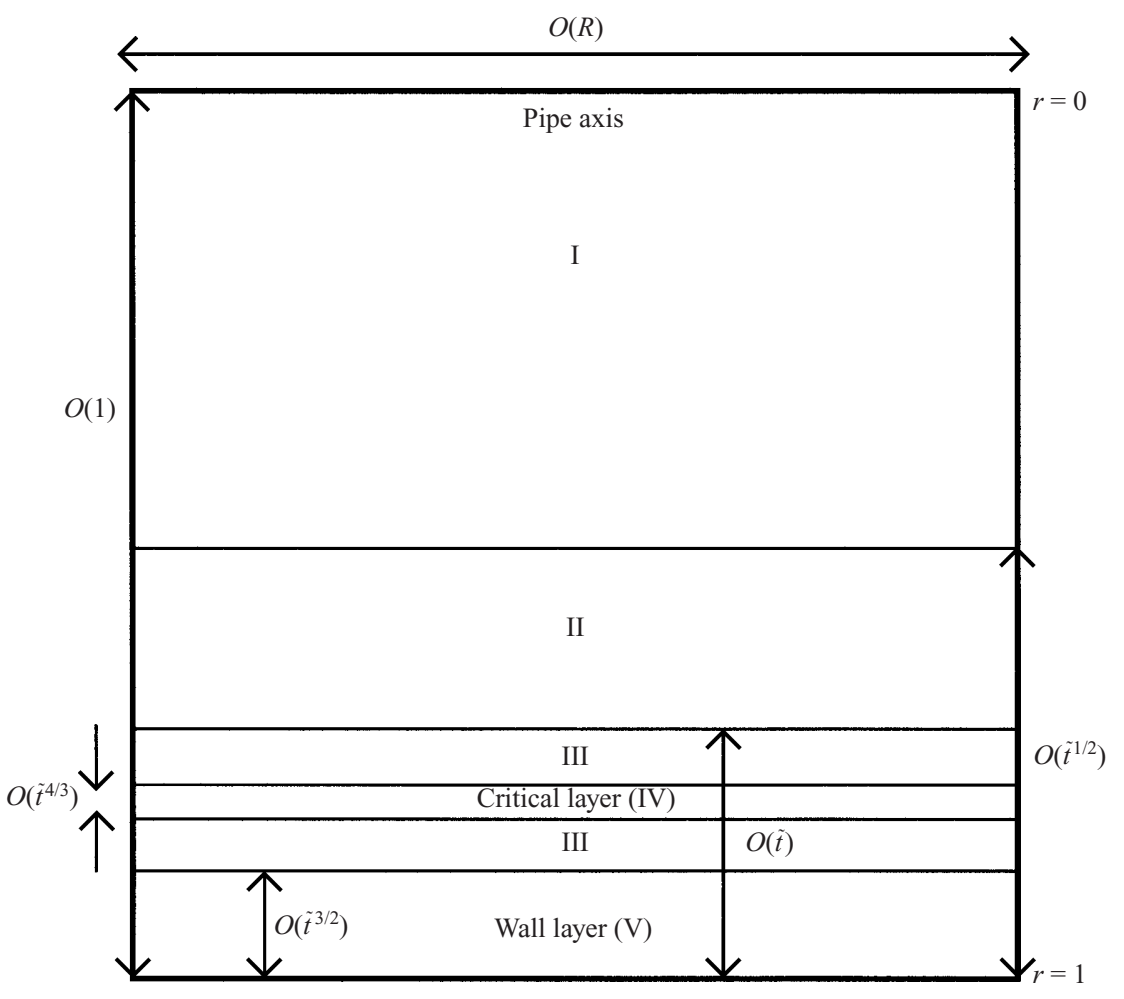

FIGURE 2. The linear neutral mode structure of $\S 2$ for $t=R \tilde{t}$ with $\tilde{t} \ll 1$.

(or a reduced form of them) numerically. Nevertheless analytic progress can still be made with this problem because as we shall see in $\S 3$, provided the perturbation amplitude $\delta$ is sufficiently large, the neutral wavenumber decreases more slowly than linear theory predicts and a multiple scales approach to the subsequent nonlinear problem is possible. In this situation it turns out that the amplitude and frequency of a disturbance of fixed wavenumber can vary over the slow time scale of the basic flow and keep the wave in neutral. Moreover, it is possible to determine the time-dependent nonlinear disturbance amplitude analytically and show that the disturbance evolves naturally into the small-wavenumber limit of the neutral mode structure found in the fully developed HPF by SB. In order to demonstrate this we need to consider the nonlinear SB structure on the $O(R)$ length and time scales implied by the linear analysis we have presented here, rather than on the $O(1)$ scales assumed in their work. In addition we need to consider a time-dependent basic flow of the form (3), rather than simply the fully developed flow that arises from it as $t \rightarrow \infty$ on the $O(R)$ time scale.

Before tackling the nonlinear evolution problem it is instructive to examine the critical layer dynamics in more detail as this is where the main changes to the flow take place as a result of the increased disturbance size.

\section{The dynamics of the linear and weakly nonlinear critical layers}

In $\S 2$ we determined the form of the linear upper branch neutral modes by balancing the phase shifts across the wall and critical layers. There we simply quoted 
the classical result that a phase shift of $-\pi$ is induced upon crossing the critical layer. In the presence of a larger disturbance the phase shift is modified (Benney \& Bergeron 1969; Haberman 1972) and becomes dependent on the amplitude of the perturbation. In this section we show specifically how this arises for our particular basic flow and how the reduced phase shift plays a key role in the subsequent nonlinear evolution of the flow. We begin by discussing the linear critical layer and then show how the dynamics are modified as the magnitude of the perturbation is increased.

\subsection{The linear critical layer}

The balance in the linear critical layer is between viscosity and the inertial operator $u_{0} \partial / \partial x$. From the scalings of region III we see that this requires the relation

$$
\left(\frac{\varepsilon R}{t_{0}}\right)^{-3 / 2} \alpha_{0} \zeta \sim \frac{1}{R} \frac{(\varepsilon R)^{2}}{t_{0}^{2} \zeta^{2}}
$$

Taking $\varepsilon=R^{-7 / 9}$ (as determined in the previous section) and rearranging we have

$$
\zeta=R^{-2 / 27} t_{0}^{-7 / 6} \alpha_{0}^{-1 / 3 \hat{\zeta}}
$$

where $\hat{\zeta}$ is the $O(1)$ normal variable within the critical layer. The appropriate radial scaling in this layer is therefore

$$
r=1-R^{-2 / 9} t_{0} \frac{\bar{c}_{0}}{\lambda_{0}}-R^{-8 / 27} t_{0}^{-1 / 6} \alpha_{0}^{-1 / 3} \frac{\hat{\zeta}}{\lambda_{0}}
$$

or equivalently in terms of the scaling in region III:

$$
Y=\frac{\bar{c}_{0}}{\lambda_{0}}+R^{-2 / 27} t_{0}^{-7 / 6} \alpha_{0}^{-1 / 3} \frac{\hat{\zeta}}{\lambda_{0}} .
$$

The disturbance velocities and pressure in the critical layer have the form

$$
\begin{gathered}
\delta \hat{u}=\delta R^{11 / 27} \alpha_{0}^{-5 / 3} t_{0}^{-1 / 3} \hat{u}_{1}(\hat{\zeta}) \cos \xi+\cdots+\delta R^{2 / 9} \alpha_{0}^{-2} t_{0}^{-1} \hat{u}_{L}(\xi, \hat{\zeta})+\cdots \\
\delta \hat{v}=-\delta R^{1 / 9} \alpha_{0}^{-1} t_{0}^{-1 / 2} \frac{\left(N^{2}+\alpha_{0}^{2}\right)}{4 \lambda_{0}} \sin \xi+\cdots-\delta R^{-2 / 27} \alpha_{0}^{-4 / 3} t_{0}^{-7 / 6} \hat{v}_{L}(\xi, \hat{\zeta})+\cdots, \\
\delta \hat{w}=\delta R^{11 / 27} \alpha_{0}^{-2 / 3} t_{0}^{-1 / 3} \hat{w}_{1}(\hat{\zeta}) \cos \xi+\cdots+\delta R^{2 / 9} \alpha_{0}^{-1} t_{0}^{-1} \hat{w}_{L}(\xi, \hat{\zeta})+\cdots \\
\delta \hat{p}=\delta \cos \xi+\cdots
\end{gathered}
$$

Here, the first terms in the expansions for $\hat{u}$ and $\hat{w}$ serve to smooth out the algebraic singularity that arises as a three-dimensional effect in the Rayleigh equation of region III. This can be seen by consideration of (9), where both $F$ and $H$ are proportional to $\left(u_{0}-c\right)^{-1}$ as $u_{0} \rightarrow c$. The terms with subscript $L$ (also present in twodimensional critical layers) form part of the continuation and regularization of the logarithmic behaviour observed in region III and will be referred to as the logarithmic contributions.

The basic flow within the critical layer is simply the Taylor expansion of its form in region III about the critical location and can be written as

$$
u_{0}=4 R^{-1 / 3} t_{0}^{3 / 2} \bar{c}_{0}+4 R^{-11 / 27} \alpha_{0}^{-1 / 3} t_{0}^{1 / 3} \hat{\zeta}+\cdots+4 R^{-16 / 27} \frac{\lambda_{1}}{\lambda_{0}^{2}} \alpha_{0}^{-2 / 3} t_{0}^{-1 / 3} \hat{\zeta}^{2}+\cdots .
$$

In both sets of expansions above we have omitted terms that do not play a vital 
role in determining the phase shift. The leading-order disturbances $\hat{u}_{1}$ and $\hat{w}_{1}$ satisfy forced Airy equations and from the continuity balance they are related via

$$
\hat{u}_{1}+N \hat{w}_{1}=0 .
$$

We now turn to the terms that smooth out the logarithmic behaviour. From substitution into the LNS equations we have the governing equations

$$
\begin{gathered}
\frac{\partial \hat{u}_{L}}{\partial \xi}+\lambda_{0} \frac{\partial \hat{v}_{L}}{\partial \hat{\zeta}}+N \frac{\partial \hat{w}_{L}}{\partial \xi}=0, \\
4 \hat{\zeta} \frac{\partial \hat{u}_{L}}{\partial \xi}+4 \lambda_{0} \hat{v}_{L}-4 \frac{\lambda_{1}}{\lambda_{0}^{2}} \hat{\zeta}^{2} \hat{u}_{1} \sin \xi+2 \frac{\lambda_{1}}{\lambda_{0}^{2}}\left(N^{2}+\alpha_{0}^{2}\right) \hat{\zeta} \sin \xi=\lambda_{0}^{2} \frac{\partial^{2} \hat{u}_{L}}{\partial \hat{\zeta}^{2}} \\
4 \hat{\zeta} \frac{\partial \hat{w}_{L}}{\partial \xi}-4 \frac{\lambda_{1}}{\lambda_{0}^{2}} \hat{\zeta}^{2} \hat{w}_{1} \sin \xi=\lambda_{0}^{2} \frac{\partial^{2} \hat{w}_{L}}{\partial \hat{\zeta}^{2}} .
\end{gathered}
$$

We define a skewed velocity and a streamfunction such that

$$
\bar{u}_{L}=\hat{u}_{L}+N \hat{w}_{L}=\frac{\partial \psi}{\partial \hat{\zeta}}, \quad \hat{v}_{L}=-\frac{1}{\lambda_{0}} \frac{\partial \psi}{\partial \xi} .
$$

Then, by combining $(37 b, c)$, using (36) and differentiating with respect to $\hat{\zeta}$ we obtain the governing critical layer equation in the linear regime:

$$
4 \hat{\zeta} \frac{\partial^{3} \psi}{\partial \xi \partial \hat{\zeta}^{2}}+2 \frac{\lambda_{1}}{\lambda_{0}^{2}}\left(N^{2}+\alpha_{0}^{2}\right) \sin \xi=\lambda_{0}^{2} \frac{\partial^{4} \psi}{\partial \hat{\zeta}^{4}} .
$$

By examining the behaviour of the logarithmic part of the term $\hat{G}_{1}$ (and the corresponding form for the streamwise perturbation) in region III we obtain the matching conditions

$$
\begin{aligned}
& \frac{\partial \psi}{\partial \hat{\zeta}} \sim \frac{\lambda_{1}}{2 \lambda_{0}^{2}}\left(N^{2}+\alpha_{0}^{2}\right)(\ln \hat{\zeta})(\cos \xi) \quad \text { as } \hat{\zeta} \rightarrow+\infty, \\
& \frac{\partial \psi}{\partial \hat{\zeta}} \sim \frac{\lambda_{1}}{2 \lambda_{0}^{2}}\left(N^{2}+\alpha_{0}^{2}\right)(\ln |\hat{\zeta}| \cos \xi-\phi \sin \xi) \quad \text { as } \hat{\zeta} \rightarrow-\infty,
\end{aligned}
$$

where $\phi$ is the anticipated phase shift across the critical layer. For the present linear critical layer the variables can be separated in (38), and asymptotic analysis (see for example Haberman 1976) shows that

$$
\phi=-\pi,
$$

as assumed in $\S 2$. This is of course consistent with earlier work on linear critical layers (e.g. Stuart 1963; Reid 1965). We have discussed the linear critical layer in more detail than usual because the scalings in operation here can be used to infer the disturbance amplitude at which the critical layer first exhibits significant nonlinear effects and the above analysis becomes invalid.

By inspection of (34a) and (35) we anticipate that the first major change to the critical layer properties arises when the leading-order streamwise perturbation becomes of the same order of magnitude as the shear contribution to the basic flow, i.e. a new stage is reached when

$$
\delta R^{11 / 27} \alpha_{0}^{-5 / 3} t_{0}^{-1 / 3} \sim R^{-11 / 27} \alpha_{0}^{-1 / 3} t_{0}^{1 / 3},
$$


implying an enhanced amplitude of

$$
\delta \sim R^{-22 / 27} \alpha_{0}^{4 / 3} t_{0}^{2 / 3}
$$

Thus it appears that a disturbance of the still tiny size $O\left(R^{-22 / 27}\right)$ is sufficient to provoke nonlinear effects within the critical layer, although the rest of the flow is altered only slightly. We now proceed to discuss precisely how the critical layer is altered for this enhanced disturbance size and in particular the implications for the amplitude dependence of the induced phase shift.

\subsection{The weakly nonlinear critical layer}

As far as the calculation of the phase shift is concerned the main change we encounter as a result of the increased disturbance size is in the streamwise velocity profile which is now a combination of the basic flow and the perturbation and has the expansion

$$
\begin{aligned}
u & =u_{0}+\delta \hat{u} \\
= & 4 R^{-1 / 3} t_{0}^{3 / 2} \bar{c}_{0}+R^{-11 / 27} \alpha_{0}^{-1 / 3} t_{0}^{1 / 3} \hat{u}_{1}(\xi, \hat{\zeta})+\cdots \\
& \quad+R^{-16 / 27} \alpha_{0}^{-2 / 3} t_{0}^{-1 / 3} \hat{u}_{L}(\xi, \hat{\zeta})+\cdots,
\end{aligned}
$$

for the enhanced disturbance size

$$
\delta=R^{-22 / 27} \alpha_{0}^{4 / 3} t_{0}^{2 / 3} \hat{A},
$$

with the amplitude parameter $\hat{A} \sim O(1)$ at present. The new critical layer balances are then determined by substituting (40) and the corresponding expansions for the other velocity components (which are largely unchanged) into the nonlinear Navier-Stokes equations (1). At leading order the continuity equation yields

$$
\frac{\partial}{\partial \xi}\left(\hat{u}_{1}+N \hat{w}_{1}\right)=0
$$

and our matching conditions require

$$
\hat{u}_{1} \sim 4 \hat{\zeta}, \quad \hat{w}_{1} \rightarrow 0 \quad \text { as } \quad \hat{\zeta} \rightarrow \infty .
$$

After some manipulation and use of (42), the leading-order momentum balances give

$$
\frac{\hat{A}}{4}\left(N^{2}+\alpha_{0}^{2}\right) \sin \xi \frac{\partial}{\partial \hat{\zeta}}\left(\hat{u}_{1}+N \hat{w}_{1}\right)=\hat{A}\left(N^{2}+\alpha_{0}^{2}\right) \sin \xi+\lambda_{0}^{2} \frac{\partial^{2}}{\partial \hat{\zeta}^{2}}\left(\hat{u}_{1}+N \hat{w}_{1}\right),
$$

which (upon use of (43)) leads to the simple result

$$
\hat{u}_{1}+N \hat{w}_{1}=4 \hat{\zeta}
$$

and this can be used to eliminate some of the inertial contributions to the equations governing the behaviour of the logarithmic terms. Using the skewed velocity and streamfunction notation (now denoted by $\Psi$ ) from earlier we can deduce that the critical layer dynamics are now controlled by the equation

$$
4 \hat{\zeta} \frac{\partial^{3} \Psi}{\partial \xi \partial \hat{\zeta}^{2}}+\frac{\hat{A}\left(N^{2}+\alpha_{0}^{2}\right)}{4} \sin \xi \frac{\partial^{3} \Psi}{\partial \hat{\zeta}^{3}}=\lambda_{0}^{2} \frac{\partial^{4} \Psi}{\partial \hat{\zeta}^{4}} .
$$

If $\hat{A} \ll 1$, the streamfunction equation can be linearized by writing $\Psi=\left(4 \lambda_{1} / 3 \lambda_{0}^{2}\right) \hat{\zeta}^{3}+$ $\hat{A} \psi$, where $\psi$ satisfies the linear equation (38) and matching conditions (39) of the previous subsection and therefore we conclude that the phase shift $\phi \rightarrow-\pi$ as $\hat{A} \rightarrow 0$. 
When $\hat{A} \sim O(1)$ however, the situation is not as straightforward and we need to draw on some two-dimensional results. The same form of equation as (45) governs the dynamics of weakly nonlinear two-dimensional critical layers and has been studied in this context by Brown \& Stewartson (1978) and Smith \& Bodonyi (1982b) among others. Their numerical studies suggest that the finite part of the velocity jump is not of the monochromatic form found in the linear critical layer and in addition there is a jump in the vorticity across the layer. These findings imply that the appropriate matching conditions to be applied to (45) take the more complicated form

$$
\begin{aligned}
& \frac{\partial \Psi}{\partial \hat{\zeta}} \sim \frac{4 \lambda_{1}}{\lambda_{0}^{2}} \hat{\zeta}^{2}+\hat{A}^{1 / 2} \hat{\lambda}^{+} \hat{\zeta}+\hat{A} \frac{\lambda_{1}}{2 \lambda_{0}^{2}}\left(N^{2}+\alpha_{0}^{2}\right)(\ln \hat{\zeta})(\cos \xi) \quad \text { as } \hat{\zeta} \rightarrow+\infty \\
& \frac{\partial \Psi}{\partial \hat{\zeta}} \sim \frac{4 \lambda_{1}}{\lambda_{0}^{2}} \hat{\zeta}^{2}+\hat{A}^{1 / 2} \hat{\lambda}^{-} \hat{\zeta}+\hat{A} \frac{\lambda_{1}}{2 \lambda_{0}^{2}}\left(N^{2}+\alpha_{0}^{2}\right)(\ln |\hat{\zeta}| \cos \xi-J(\xi)) \quad \text { as } \hat{\zeta} \rightarrow-\infty .
\end{aligned}
$$

Here, the velocity jump $J(\xi)$ is determined as part of the solution, the constant $\hat{\lambda}^{+}-\hat{\lambda}^{-}$represents the jump in vorticity across the layer, while the term proportional to $\hat{\zeta}^{2}$ arises from the quadratic part of the basic flow. These new conditions can be reconciled with the flow in region III which is slightly modified as a result. Although the velocity jump is no longer monochromatic the concept of a phase shift is still valid provided we define $\phi$ to be the coefficient of $\sin \xi$ in the Fourier series expansion of $J(\xi)$, i.e.

$$
\phi=\frac{1}{N \pi} \int_{0}^{2 N \pi} J(\xi) \sin \xi \mathrm{d} \xi
$$

By taking this as our definition of $\phi$ we retain the property that terms in region III of the form

$$
\ln \zeta \text { for } \zeta>0 \text { are replaced by } \ln |\zeta|+\mathrm{i} \phi \text { for } \zeta<0 \text {. }
$$

The vorticity and velocity jumps anticipated in the matching conditions (46) can be seen to be related by integrating the critical layer equation (45) twice with respect to $\hat{\zeta}$, once with respect to $\xi$ from 0 to $2 N \pi$ and then taking finite parts as $\zeta \rightarrow \pm \infty$ to obtain

$$
\frac{\hat{A}\left(N^{2}+\alpha_{0}^{2}\right)}{4} \int_{0}^{2 N \pi}[[\partial \Psi / \partial \hat{\zeta}]]_{-\infty}^{+\infty} \sin \xi \mathrm{d} \xi=\lambda_{0}^{2} \int_{0}^{2 N \pi}\left[\left[\partial^{2} \Psi / \partial \hat{\zeta}^{2}\right]\right]_{-\infty}^{+\infty} \mathrm{d} \xi
$$

where [[] ] denotes the finite part of the jump. Upon use of the matching conditions and the definition of $\phi$ we obtain

$$
\frac{\lambda_{1} \hat{A}^{2}\left(N^{2}+\alpha_{0}^{2}\right)^{2}}{8 \lambda_{0}^{2}} N \pi \phi=\lambda_{0}^{2} 2 N \pi \hat{A}^{1 / 2}\left(\hat{\lambda}^{+}-\hat{\lambda}^{-}\right),
$$

and hence

$$
\phi=\frac{16 \lambda_{0}^{4}}{\lambda_{1}\left(N^{2}+\alpha_{0}^{2}\right)^{2}}\left(\hat{\lambda}^{+}-\hat{\lambda}^{-}\right) \hat{A}^{-3 / 2}
$$

determines the phase shift in terms of the disturbance amplitude and the vorticity jump. Outside the critical layer the main change is expressed by the replacement (47). In view of this the only alteration to the linear neutral stability results derived in $\S 2$ is that the phase shift is $\phi$ rather than $-\pi$, and so, in particular, the large-time 
behaviour of the wavenumber is now given by

$$
\alpha_{0} \sim \frac{\lambda_{0}^{11}}{32 N^{5} \lambda_{1}^{2}} \frac{t_{0}^{-9 / 2}}{\phi^{2}} \quad \text { as } \quad t_{0} \rightarrow \infty,
$$

which is to be compared with the linear result (32). One way of interpreting (49) is that a wave of fixed wavelength may remain in neutral provided its amplitude $\hat{A}$ adjusts over the time scale of the developing flow so that the right-hand side of (49) remains constant.

The numerical results of Smith \& Bodonyi (1982b) indicate that $-\phi$ decreases monotonically from its linear value of $\pi$ as $\hat{A}$ increases, with $\phi \rightarrow 0$ as $\hat{A} \rightarrow \infty$. The significance of this result is that if $\hat{A} \gg 1$ we have $\phi \ll 1$ and so in the fully developed regime wherein $t_{0} \sim O\left(R^{2 / 9}\right)$ we have $\alpha_{0} \sim O\left(R^{-1} \phi^{-2}\right) \gg O\left(R^{-1}\right)$. Thus the wavelength of the instability is much less than the $O(R)$ length scale over which the mean flow develops, indicating that the subsequent evolution of the neutral disturbance, although fully nonlinear, can be analysed by continuing our asymptotic approach, and that recourse to a full numerical simulation is unnecessary. Another way of expressing this is that since the wavelength of the wave is less than $O(R)$, then if such a wave is introduced into the flow at a distance of $O(R)$ along the pipe it will not be aware of the pipe entrance and as a consequence spatial derivatives of the underlying basic flow can be neglected when considering the disturbance evolution.

Before we can consider the fully developed regime any further we need to analyse the streamfunction equation (45) for $\hat{A} \gg 1$, determine the vorticity jump explicitly and hence find the relationship between $\phi$ and $\hat{A}$. Fortunately this step has been performed already for a two-dimensional flow by Smith \& Bodonyi $(1982 b)$ and the work we present here requires only a slight modification of their original analysis.

\subsubsection{The solution of the critical layer problem at large disturbance amplitude}

When $\hat{A}$ is large, the inertia terms dominate the viscous effects in (45) and so it is convenient to write the streamfunction equation in the form

$$
\zeta^{*} \frac{\partial^{3} \Psi}{\partial \xi \partial \zeta^{* 2}}+\beta \sin \xi \frac{\partial^{3} \Psi}{\partial \zeta^{* 3}}=\frac{\lambda_{0}^{2}}{4} \hat{A}^{-3 / 2} \frac{\partial^{4} \Psi}{\partial \zeta^{* 4}},
$$

where

$$
\hat{\zeta}=\hat{A}^{1 / 2 \zeta^{*}}, \quad \beta=\frac{N^{2}+\alpha_{0}^{2}}{16} .
$$

The solution at large $\hat{A}$ then develops as

$$
\frac{\partial^{2} \Psi}{\partial \zeta^{* 2}}=\hat{A}^{3 / 2} \chi_{0}+\chi_{1}+\cdots,
$$

where the leading-order contribution satisfies the homogeneous version of (50) and has the solution

$$
\chi_{0}=\mathscr{G}(\hat{\eta}), \quad \hat{\eta}=\frac{1}{2} \zeta^{* 2}+\beta \cos \xi .
$$

This is an expression of the conservation of vorticity. The streamlines here are of cat's-eye form and are closed for $\hat{\eta}<\beta$. Application of the Prandtl-Batchelor theorem leads to the result

$$
\mathscr{G}(\hat{\eta})=\mathscr{G}_{0} \quad \text { for } \hat{\eta}<\beta
$$


where $\mathscr{G}_{0}$ is an unknown constant. At the next order the correction $\chi_{1}$ satisfies

$$
\zeta^{*} \frac{\partial \chi_{1}}{\partial \xi}+\beta \sin \xi \frac{\partial \chi_{1}}{\partial \zeta^{*}}=\frac{\lambda_{0}^{2}}{4} \frac{\partial^{2} \chi_{0}}{\partial \zeta^{* 2}}
$$

Changing to characteristic coordinates and substituting for $\chi_{0}$ we obtain

$$
\pm \frac{\partial \chi_{1}}{\partial \hat{\xi}}=\frac{\lambda_{0}^{2}}{2 \sqrt{2}} \frac{\partial}{\partial \hat{\eta}}\left((\hat{\eta}-\beta \cos \hat{\xi})^{1 / 2} \mathscr{G}^{\prime}(\hat{\eta})\right),
$$

where the new independent variables are $\hat{\xi}$ and $\hat{\eta}$ with $\xi=\hat{\xi}$. Here the plus and minus signs refer to the upper and lower parts of the critical layer where $\zeta^{*}>$ $(2 \beta)^{1 / 2}(1-\cos \hat{\xi})^{1 / 2}$ and $\zeta^{*}<-(2 \beta)^{1 / 2}(1-\cos \hat{\xi})^{1 / 2}$, respectively.

In view of the wave-like form of the disturbances, the quantity $\chi_{1}$ must be periodic in $\hat{\xi}$ with period $2 N \pi$. Integrating (51) over this range and applying periodicity we find that

$$
\mathscr{G}^{\prime}(\hat{\eta})=\frac{D^{ \pm}}{\hat{I}(\hat{\eta})}, \quad \hat{I}(\hat{\eta})=\int_{0}^{2 N \pi}(\hat{\eta}-\beta \cos q)^{1 / 2} \mathrm{~d} q .
$$

The constants $D^{ \pm}$are determined by the matching with region III, which in terms of the new variables may be written

$$
\frac{\partial^{2} \Psi}{\partial \zeta^{* 2}} \sim \hat{A}^{3 / 2}\left(\frac{8 \lambda_{1}}{\lambda_{0}^{2}} \zeta^{*}+\hat{\lambda}^{ \pm}+O\left(\frac{1}{\zeta^{*}}\right)\right) \text { as } \zeta^{*} \rightarrow \pm \infty,
$$

from (46). The values of $D^{ \pm}$are therefore fixed as

$$
D^{ \pm}= \pm \frac{8 N \pi \sqrt{2} \lambda_{1}}{\lambda_{0}^{2}}
$$

and the leading-order solution takes the form

$$
\chi_{0}=\mathscr{G}(\hat{\eta})=\mathscr{G}_{0} \pm \frac{8 N \pi \sqrt{2} \lambda_{1}}{\lambda_{0}^{2}} \int_{\beta}^{\hat{\eta}} \frac{\mathrm{d} s}{\hat{I}(s)},
$$

where we have assumed continuity of vorticity at the edge of the cat's eye (see Brown \& Stewartson 1978). Examining the matching condition (53) a second time we see that the vorticity jump is given by

$$
\hat{\lambda}^{+}-\hat{\lambda}^{-}=\left[\left[\chi_{0}\right]\right]_{-\infty}^{+\infty}=\frac{16 N \pi \sqrt{2} \lambda_{1}}{\lambda_{0}^{2}} f_{\beta}^{\infty} \frac{\mathrm{d} s}{\hat{I}(s)},
$$

where [[]] denotes the finite part of the jump and the bar denotes the finite part of the integral, the value of which can be computed to be

$$
f_{\beta}^{\infty} \frac{\mathrm{d} s}{\hat{I}(s)}=\frac{(2 \beta)^{1 / 2}}{8 \pi N} C^{(1)}, \quad C^{(1)} \simeq-5.516 .
$$

This integral has arisen in a number of previous critical layer studies (we will meet it again in $\S 4$ ) and its value has been computed previously by Haberman (1976) and Smith \& Bodonyi (1982b), among others. It is now possible to determine the amplitude dependence of the phase shift by use of (48) and we find

$$
\phi=\frac{16 \lambda_{0}^{2} C^{(1)}}{\left(N^{2}+\alpha_{0}^{2}\right)^{3 / 2}} \hat{A}^{-3 / 2} .
$$


Thus the main effect of increasing the disturbance size is to decrease the phase shift and this is particularly significant as we move into the fully developed regime which we consider next.

\subsubsection{The large-amplitude weakly nonlinear critical layer structure at larger times}

Now that we have determined the amplitude dependence of the phase shift (54) we can rewrite the large-time neutral wavenumber result (49) as

$$
\alpha_{0} \sim \frac{N \lambda_{0}^{7}}{2^{13} \lambda_{1}^{2}\left(C^{(1)}\right)^{2}} \hat{A}^{3} t_{0}^{-9 / 2} \text { as } t_{0} \rightarrow \infty .
$$

Thus in the fully developed regime the leading-order wavenumber dependence may be written as

$$
\alpha_{0} \sim R^{-1} \tilde{\alpha}, \quad \text { with } \quad \tilde{\alpha}=\frac{N \lambda_{0}^{7}}{2^{13} \lambda_{1}^{2}\left(C^{(1)}\right)^{2}} \hat{A}^{3} \tilde{t}^{-9 / 2} \gg 1,
$$

and $\tilde{t}=R^{2 / 9} t_{0}$ is the $O(1)$ temporal variable in the fully developed regime.

The scaling (41) of the disturbance size $\delta$ suggests that as time increases the perturbation will increase in amplitude and nonlinear effects will become even more significant within the critical layer. In order to provide us with a clue to the form the scalings will take in the fully developed regime it is instructive to write $\delta$ and the induced phase shift $\phi$ in terms of the new variables $\tilde{\alpha}$ and $\tilde{t}$. We then find from (41), (54) and (55)

$$
\delta \sim R^{-2} \tilde{\alpha}^{5 / 3} \frac{2^{13 / 3}\left(\lambda_{1} C^{(1)}\right)^{2 / 3}}{N^{1 / 3} \lambda_{0}^{7 / 3}} \tilde{t}^{13 / 6}, \quad \phi \sim \tilde{\alpha}^{-1 / 2} \frac{\lambda_{0}^{11 / 2}}{(2 N)^{5 / 2} \lambda_{1}} \tilde{t}^{-9 / 4},
$$

as $\tilde{t} \rightarrow 0$. This suggests that in the strongly nonlinear fully developed regime we should seek solutions with a neutral wavenumber of order $R^{-1} \tilde{\alpha}$ and an $O\left(R^{-2} \tilde{\alpha}^{5 / 3}\right)$ pressure disturbance amplitude, leading to an induced $O\left(\tilde{\alpha}^{-1 / 2}\right)$ phase shift, where the parameter $\tilde{\alpha}$ is large and is given specifically in (55). This provides the motivation for the nonlinear study to be considered in the next section.

\section{Nonlinear analysis}

Our findings from the previous section indicate that in the fully developed regime where $t \sim O(R)$, the disturbance has an $O\left(R^{-2} \tilde{\alpha}^{5 / 3}\right)$ pressure amplitude and an $O\left(R^{-1} \tilde{\alpha}\right)$ wavenumber where $\tilde{\alpha} \gg 1$. Considering the Reynolds number dependence first we write

$$
\frac{\partial}{\partial t}=R^{-1} \frac{\partial}{\partial \tilde{t}}, \quad \frac{\partial}{\partial x}=R^{-1} \frac{\partial}{\partial \tilde{x}},
$$

and we scale the velocities and pressure in the form

$$
\begin{gathered}
u=\tilde{u}(r, \theta, \tilde{x}, \tilde{t})+\cdots, \quad v=R^{-1} \tilde{v}(r, \theta, \tilde{x}, \tilde{t})+\cdots, \\
w=R^{-1} \tilde{w}(r, \theta, \tilde{x}, \tilde{t})+\cdots, \quad p=p_{0}-R^{-1} 4 x+R^{-2} \tilde{p}(r, \theta, \tilde{x}, \tilde{t})+\cdots .
\end{gathered}
$$

Substitution of these expansions into the Navier-Stokes equations (1) leads to the following nonlinear viscous balances

$$
\frac{\partial \tilde{u}}{\partial \tilde{x}}+\frac{\partial \tilde{v}}{\partial r}+\frac{\tilde{v}}{r}+\frac{1}{r} \frac{\partial \tilde{w}}{\partial \theta}=0,
$$




$$
\begin{gathered}
\frac{\partial \tilde{u}}{\partial \tilde{t}}+\tilde{u} \frac{\partial \tilde{u}}{\partial \tilde{x}}+\tilde{v} \frac{\partial \tilde{u}}{\partial r}+\frac{\tilde{w}}{r} \frac{\partial \tilde{u}}{\partial \theta}=4+\frac{\partial^{2} \tilde{u}}{\partial r^{2}}+\frac{1}{r} \frac{\partial \tilde{u}}{\partial r}+\frac{1}{r^{2}} \frac{\partial^{2} \tilde{u}}{\partial \theta^{2}}, \\
\frac{\partial \tilde{v}}{\partial \tilde{t}}+\tilde{u} \frac{\partial \tilde{v}}{\partial \tilde{x}}+\tilde{v} \frac{\partial \tilde{v}}{\partial r}+\frac{\tilde{w}}{r} \frac{\partial \tilde{v}}{\partial \theta}-\frac{\tilde{w}^{2}}{r} \\
=-\frac{\partial \tilde{p}}{\partial r}+\frac{\partial^{2} \tilde{v}}{\partial r^{2}}+\frac{1}{r} \frac{\partial \tilde{v}}{\partial r}+\frac{1}{r^{2}} \frac{\partial^{2} \tilde{v}}{\partial \theta^{2}}-\frac{\tilde{v}}{r^{2}}-\frac{2}{r^{2}} \frac{\partial \tilde{w}}{\partial \theta}, \\
\frac{\partial \tilde{w}}{\partial \tilde{t}}+\tilde{u} \frac{\partial \tilde{w}}{\partial \tilde{x}}+\tilde{v} \frac{\partial \tilde{w}}{\partial r}+\frac{\tilde{w}}{r} \frac{\partial \tilde{w}}{\partial \theta}+\frac{\tilde{v} \tilde{w}}{r} \\
=-\frac{1}{r} \frac{\partial \tilde{p}}{\partial \theta}+\frac{\partial^{2} \tilde{w}}{\partial r^{2}}+\frac{1}{r} \frac{\partial \tilde{w}}{\partial r}+\frac{1}{r^{2}} \frac{\partial^{2} \tilde{w}}{\partial \theta^{2}}-\frac{\tilde{w}}{r^{2}}+\frac{2}{r^{2}} \frac{\partial \tilde{v}}{\partial \theta},
\end{gathered}
$$

which we will refer to as the reduced Navier-Stokes equations (RNS). Note that the Reynolds number does not appear in these equations and that one possible solution of the system is simply the HPF profile $\tilde{u}=1-r^{2}, \tilde{v}=\tilde{w}=0$. The RNS system (58) will act as our governing equations for the rest of the section. Now taking into account the $\tilde{\alpha}$-dependence, we seek disturbances proportional to

$$
\operatorname{expi} \tilde{\xi},
$$

with $\tilde{\xi}=\tilde{\alpha}(\tilde{x}-c \tilde{t})+N \theta$, and $\tilde{\alpha} \gg 1$, where the pressure perturbation has amplitude

$$
\tilde{\alpha}^{5 / 3} A_{0}(\tilde{t}) \text {. }
$$

We will assume that the wave speed $c \sim O(1)$ for $O(1)$ values of $\tilde{t}$ and we write $c=c_{0}$ to leading order. In view of the results obtained for large $t_{0}$ in the previous section we anticipate that for small $\tilde{t}$

$$
A_{0} \sim \frac{2^{13 / 3}\left(\lambda_{1} C^{(1)}\right)^{2 / 3}}{N^{1 / 3} \lambda_{0}^{7 / 3}} \tilde{t}^{13 / 6}, \quad c_{0} \sim \frac{4 N}{\lambda_{0}} \tilde{t}^{3 / 2},
$$

from $(33),(56 a, b)$. We will see later that these asymptotes are indeed obtained in the small- $\tilde{t}$ limit of the current nonlinear problem: for now we concentrate on $\tilde{t} \sim O(1)$. The basic flow remains the impulsively started flow given in $\S 1$ and has the form

$$
\tilde{u}_{0}=1-r^{2}-8 \sum_{n=1}^{\infty} \frac{J_{0}\left(j_{o n} r\right)}{j_{o n}^{3} J_{1}\left(j_{o n}\right)} \exp \left(-j_{o n}^{2} \tilde{t}\right)
$$

in the current notation. Since we are now considering the development at $O(1)$ times, the basic flow can no longer be written in terms of the two-tier structure (4) that was exploited in the previous sections. Nevertheless it still proves possible to study analytically the nonlinear stability of $\tilde{u}_{0}$ to the long waves introduced above even when $\tilde{u}_{0}$ takes the above more complicated form (60).

For $\tilde{t} \sim O(1)$, the stability structure consists of three main regions. In the majority of the pipe the flow is predominantly inviscid and the behaviour is similar to that in region I of the linear stability structure. Since we are assuming that the wave speed is $O(1)$ however, the critical layer is no longer sited near the wall. As in the linear stability structure we require a wall layer in order both to satisfy the no-slip condition and to balance the phase shift across the critical layer which, as we shall see later, is asymptotically small (specifically of $O\left(\tilde{\alpha}^{-1 / 2}\right)$, as predicted in $\S 3$ ), in the nonlinear setting considered here. 


\subsection{The inviscid region}

We begin by considering the flow in the majority of the pipe where the radial variable $r$ is $O(1)$. Since the pressure amplitude is known, the magnitude of the velocity perturbations can also be determined and the appropriate expansions are

$$
\begin{gathered}
\tilde{u}=\tilde{u}_{0}(r, \tilde{t})+\tilde{\alpha}^{-1 / 6} u_{1 M}(r, \tilde{t})+\tilde{\alpha}^{-1 / 3}\left(\tilde{u}_{2}(r, \theta, \tilde{x}, \tilde{t})+\tilde{u}_{2 M}(r, \tilde{t})\right) \\
+\cdots+\tilde{\alpha}^{-5 / 6} \tilde{u}_{5}(r, \theta, \tilde{x}, \tilde{t})+\cdots, \\
\tilde{v}=\tilde{\alpha}^{2 / 3} \tilde{v}_{2}(r, \theta, \tilde{x}, \tilde{t})+\cdots+\tilde{\alpha}^{1 / 6} \tilde{v}_{5}(r, \theta, \tilde{x}, \tilde{t})+\cdots, \\
\tilde{w}=\tilde{\alpha}^{5 / 6} w_{1 M}(r, \tilde{t})+\tilde{\alpha}^{2 / 3}\left(\tilde{w}_{2}(r, \theta, \tilde{x}, \tilde{t})+\tilde{w}_{2 M}(r, \tilde{t})\right)+\cdots+\tilde{\alpha}^{1 / 6} \tilde{w}_{5}(r, \theta, \tilde{x}, \tilde{t})+\cdots, \\
\tilde{p}=\tilde{\alpha}^{5 / 3}\left(\tilde{p}_{2}(r, \theta, \tilde{x}, \tilde{t})+\tilde{p}_{2 M}(r, \tilde{t})\right)+\cdots+\tilde{\alpha}^{7 / 6} \tilde{p}_{5}(r, \theta, \tilde{x}, \tilde{t})+\cdots,
\end{gathered}
$$

with $\tilde{\alpha} \gg 1$. The subscript $M$ refers to a mean flow correction whose size can be inferred from the vorticity jump in the weakly nonlinear critical layer of $\S 3$. The appearance of the terms with subscript 5 anticipate the occurrence of the induced $O\left(\tilde{\alpha}^{-1 / 2}\right)$ phase shift which we will discuss in more detail later. The fundamental disturbances $\left(\tilde{u}_{2}, \tilde{v}_{2}, \tilde{w}_{2}, \tilde{p}_{2}\right)$ take the form of the real parts of

$$
A_{0}(\tilde{t})\left(F_{2}(r), \mathrm{i} G_{2}(r), H_{2}(r), Q_{2}(r)\right) \exp (\mathrm{i} \tilde{\xi})
$$

respectively. One of the aims of the analysis is to determine the real amplitude $A_{0}$ of the neutral modes and its dependence upon $\tilde{t}$ and to confirm the asymptotic results $(59 a, b)$. In the process the phase shift across the critical layer will be calculated and we will show that its small- $\tilde{t}$ form is in accordance with $(56 a, b)$. As in the linear analysis, because $\tilde{\alpha} c \gg 1 / \tilde{t}$, the time $\tilde{t}$ can be considered purely as a parameter in the problem and derivatives with respect to this parameter can be neglected to the order to which we work. Consequently the $\tilde{t}$-dependence in the mean flow terms of (61) will be suppressed in what follows. As remarked above we take the wave speed to be $O(1)$ and write

$$
c=c_{0}+O\left(\tilde{\alpha}^{-1 / 2}\right) .
$$

As part of the analysis we will determine the leading-order wave speed $c_{0}$ of the nonlinear neutral modes. We expect that as $\tilde{t} \rightarrow 0, c_{0}$ will decrease according to $(59 a, b b)$.

We substitute the expansions (61), (62), (63) into the governing equations (58) and obtain

$$
\begin{gathered}
F_{2}+G_{2}^{\prime}+\frac{G_{2}}{r}+\frac{N H_{2}}{r}=0, \quad\left(\tilde{u}_{0}-c_{0}\right) F_{2}+G_{2} \tilde{u}_{0}^{\prime}=0, \\
\left(\tilde{u}_{0}-c_{0}\right) G_{2}=Q_{2}^{\prime}, \quad\left(\tilde{u}_{0}-c_{0}\right) H=-\frac{N Q_{2}}{r} .
\end{gathered}
$$

These equations should be compared with the inviscid balances (9) occurring in the linear structure of $\S 2$. As explained in that section we can eliminate $F_{2}, G_{2}, H_{2}$ and derive an equation solely for the pressure:

$$
\left(\tilde{u}_{0}-c_{0}\right)\left(Q_{2}^{\prime \prime}+\frac{1}{r} Q_{2}^{\prime}-\frac{N^{2}}{r^{2}} Q_{2}\right)=2 \tilde{u}_{0}^{\prime} Q_{2}^{\prime} .
$$

Observe that this is effectively the Rayleigh equation derived in $\S 2$ with $\alpha_{0}=0$ : we will refer to it as the reduced Rayleigh equation. The appropriate boundary conditions are (assuming $N \neq 0$ )

$$
Q_{2}=0 \quad \text { on } \quad r=0, \quad Q_{2}^{\prime}=0 \quad \text { on } \quad r=1 .
$$


The first condition prevents the occurrence of a singularity on the pipe axis $r=0$, while the second provides the inviscid condition of tangential flow at the wall (as in the linear case). Clearly we can see from (64) that the velocity components are singular at the radial location where $\tilde{u}_{0}=c_{0}$. We define $r_{c}$ such that

$$
\tilde{u}_{0}=c_{0} \quad \text { when } \quad r=r_{c} \text {, }
$$

and note that $r_{c}$ depends on $\tilde{t}$. We calculate from (64) and (65) that as $r \rightarrow r_{c}-$

$$
\begin{aligned}
& F_{2} \sim \frac{N^{2} \hat{p}}{r_{c}^{2} \tau_{0}}\left(\frac{1}{\tilde{\varepsilon}}-\left(1-\frac{2 \tau_{1}}{\tau_{0}}\right) \ln \tilde{\varepsilon}-\frac{3 j_{3}}{N^{2}}-\frac{1}{3}\left(1-\frac{2 \tau_{1}}{\tau_{0}}\right)\right)+O(\tilde{\varepsilon} \ln \tilde{\varepsilon}), \\
& G_{2} \sim \frac{N^{2} \hat{p}}{r_{c} \tau_{0}}\left(1-\left(1-\frac{2 \tau_{1}}{\tau_{0}}\right) \tilde{\varepsilon} \ln \tilde{\varepsilon}-\left(\frac{1}{3}+\frac{3 j_{3}}{N^{2}}+\frac{1}{3} \frac{\tau_{1}}{\tau_{0}}\right) \tilde{\varepsilon}\right. \\
& \left.-\left(1-\frac{\tau_{1}}{\tau_{0}}-2\left(\frac{\tau_{1}}{\tau_{0}}\right)^{2}\right) \tilde{\varepsilon}^{2} \ln \tilde{\varepsilon}\right)+O\left(\widetilde{\varepsilon}^{2}\right), \\
& H_{2} \sim-\frac{N \hat{p}}{r_{c} \tau_{0}}\left(\frac{1}{\tilde{\varepsilon}}+\left(1-\frac{\tau_{1}}{\tau_{0}}\right)\right)+O(\tilde{\varepsilon}), \\
& Q_{2} \sim \hat{p}\left(1-\frac{N^{2}}{2} \tilde{\varepsilon}^{2}+\frac{N^{2}}{3}\left(1-\frac{2 \tau_{1}}{\tau_{0}}\right) \tilde{\varepsilon}^{3} \ln \tilde{\varepsilon}+j_{3} \tilde{\varepsilon}^{3}\right. \\
& \left.+\frac{N^{2}}{3}\left(1-\frac{2 \tau_{1}}{\tau_{0}}\right)\left(\frac{3}{4}+\frac{3}{2} \frac{\tau_{1}}{\tau_{0}}\right) \tilde{\varepsilon}^{4} \ln \tilde{\varepsilon}\right)+O\left(\tilde{\varepsilon}^{4}\right),
\end{aligned}
$$

where the small parameter $\tilde{\varepsilon}$ and the skin-friction and curvature coefficients $\tau_{0}, \tau_{1}$ are defined by

$$
\tilde{\varepsilon}=\frac{r_{c}-r}{r_{c}}, \quad \tau_{0}=-r_{c} \tilde{u}_{0}^{\prime}\left(r_{c}\right), \quad \tau_{1}=\frac{1}{2} r_{c}^{2} \tilde{u}_{0}^{\prime \prime}\left(r_{c}\right),
$$

and $\hat{p}=Q_{2}\left(r_{c}\right)$. The quantity $j_{3}$ is unknown but can be found numerically by solving the reduced Rayleigh equation (65) subject to the boundary conditions (66). In addition a jump condition is required across the critical layer arising from the phase shift, which we write in the form $\tilde{\alpha}^{-1 / 2} \Phi$ where $\Phi$ is an $O(1)$ quantity to be determined. The implication of this is that terms of the form

$$
\operatorname{Re}\left(\ln \left(r_{c}-r\right) \exp (\mathrm{i} \tilde{\xi})\right) \text { for } r<r_{c}
$$

are replaced by

$$
\operatorname{Re}\left(\left(\ln \left(r-r_{c}\right)+\mathrm{i} \tilde{\alpha}^{-1 / 2} \Phi\right) \exp (i \tilde{\xi})\right) \quad \text { for } \quad r>r_{c} .
$$

In other words, $\ln \left(r_{c}-r\right) \cos \tilde{\xi}$ is replaced by $\ln \left(r-r_{c}\right) \cos \tilde{\xi}-\tilde{\alpha}^{-1 / 2} \Phi \sin \tilde{\xi}$ as we cross the critical layer. We therefore see by comparison with the expansions (61) that $\left(\tilde{u}_{5}, \tilde{v}_{5}, \tilde{w}_{5}, \tilde{p}_{5}\right)$ will be the lowest-order terms to undergo a non-zero phase shift. As far as the solution of the reduced Rayleigh equation (65) is concerned, the appropriate jump condition is simply that $Q_{2}$ remains real as the critical layer is crossed. The solution of (65) subject to the boundary conditions (66) and the jump condition will determine the value of $c_{0}$ for given values of $N$ and $\tilde{t}$. In general a numerical solution is necessary and we will return to this later in $\S 5$. Next we investigate the dynamics of the critical layer with the aim of determining the scaled phase shift $\Phi$ analytically in terms of the disturbance amplitude $A_{0}$. 


\subsection{The nonlinear critical layer}

The analysis in this section follows closely the work of SB and we adopt a similar notation to theirs wherever possible. It should be stressed however that the results presented here are for a more complicated basic flow and cannot simply be extracted from their work.

We observe from the inviscid region that as we approach the critical layer the fundamental azimuthal disturbance $\sim \tilde{\alpha}^{2 / 3} \tilde{\varepsilon}^{-1}$, while the pressure $\sim \tilde{\alpha}^{5 / 3}$. In the critical layer we balance nonlinearity against pressure gradients, with viscosity now being a higher-order effect, and so we have the balance $\tilde{p} \sim \tilde{w}^{2}$ from the azimuthal momentum equation (58d). This implies that $\tilde{\varepsilon} \sim \tilde{\alpha}^{-1 / 6}$ in the critical layer and so we write

$$
r=r_{c}+\tilde{\alpha}^{-1 / 6} Y \quad \text { with } \quad Y \sim O(1) .
$$

The velocities and pressure expand as follows:

$$
\begin{aligned}
& \tilde{u}=c_{0}+\tilde{\alpha}^{-1 / 6} U_{1}(Y, \tilde{\xi})+\left(\tilde{\alpha}^{-1 / 3} \ln \tilde{\alpha}^{-1 / 6}\right) U_{3 / 2}(Y, \tilde{\xi})+\tilde{\alpha}^{-1 / 3} U_{2}(Y, \tilde{\xi}) \\
& +\cdots+\tilde{\alpha}^{-5 / 6} U_{5}(Y, \tilde{\xi})+\cdots, \\
& \tilde{v}=\tilde{\alpha}^{2 / 3} V_{1}(Y, \tilde{\xi})+\left(\tilde{\alpha}^{1 / 2} \ln \alpha^{-1 / 6}\right) V_{3 / 2}(Y, \tilde{\xi})+\tilde{\alpha}^{1 / 2} V_{2}(Y, \tilde{\xi}) \\
& +\cdots+V_{5}(Y, \tilde{\xi})+\cdots, \\
& \tilde{w}=\tilde{\alpha}^{5 / 6} W_{1}(Y, \tilde{\xi})+\left(\tilde{\alpha}^{2 / 3} \ln \tilde{\alpha}^{-1 / 6}\right) W_{3 / 2}(Y, \tilde{\xi})+\tilde{\alpha}^{2 / 3} W_{2}(Y, \tilde{\xi}) \\
& +\cdots+\tilde{\alpha}^{1 / 6} W_{5}(Y, \tilde{\xi})+\cdots, \\
& \tilde{p}=\tilde{\alpha}^{5 / 3} P_{1}(Y, \tilde{\xi})+\left(\tilde{\alpha}^{3 / 2} \ln \tilde{\alpha}^{-1 / 6}\right) P_{3 / 2}(Y, \tilde{\xi})+\tilde{\alpha}^{3 / 2} P_{2}(Y, \tilde{\xi}) \\
& +\cdots+\tilde{\alpha} P_{5}(Y, \tilde{\xi})+\cdots
\end{aligned}
$$

Substitution of these expansions into the RNS equations (58) yields the following leading-order nonlinear balances:

$$
\begin{gathered}
U_{1 \tilde{\xi}}+V_{1 Y}+\frac{N W_{1 \tilde{\xi}}}{r_{c}}=0, \\
U_{1} U_{1 \tilde{\xi}}+V_{1} U_{1 Y}+\frac{N W_{1} U_{1 \tilde{\xi}}}{r_{c}}=0, \quad P_{1 Y}=0, \\
U_{1} W_{1 \tilde{\xi}}+V_{1} W_{1 Y}+\frac{N W_{1} W_{1 \tilde{\xi}}}{r_{c}}=-\frac{N P_{1 \tilde{\xi}}}{r_{c}} .
\end{gathered}
$$

Thus the main pressure disturbance is constant throughout the layer and assumes the value

$$
P_{1}=A_{0} \hat{p} \cos \tilde{\xi}+\tilde{p}_{2 M}\left(r_{c}\right),
$$

from (61), (62) and (67). The appropriate boundary conditions on the velocities in order to match with the inviscid regions either side of the critical layer can be deduced from the asymptotic behaviour of (67). These take the form

$$
\begin{gathered}
U_{1} \sim-\frac{\tau_{0}}{r_{c}} Y+\tilde{u}_{1 M}\left(r_{c} \pm\right)-\frac{A_{0} \hat{p} N^{2}}{r_{c} \tau_{0} Y} \cos \tilde{\xi}, \\
V_{1} \sim-\frac{A_{0} \hat{p} N^{2}}{r_{c} \tau_{0}} \sin \tilde{\xi}, \quad W_{1} \sim \frac{A_{0} \hat{p} N}{\tau_{0} Y} \cos \tilde{\xi}+\tilde{w}_{1 M}\left(r_{c} \pm\right),
\end{gathered}
$$


as $Y \rightarrow \pm \infty$, with the first term in (72a) arising from the basic flow. As may be anticipated by comparison with the weakly nonlinear critical layer, this leading-order problem can be solved analytically and this paves the way for the higher-order contributions and in particular the phase shift to be determined. The solution is of the 'cat's-eye' form:

$$
U_{1}=-\frac{N}{r_{c}} G(\tilde{\eta}), \quad V_{1}=-\tilde{\beta} \sin \tilde{\xi}, \quad W_{1}=-\frac{\tau_{0}}{N}\left(Y-\frac{r_{c} b}{\tau_{0}}\right)+G(\tilde{\eta}),
$$

cf. (44). Here we use the notation

$$
\tilde{\beta}=\frac{A_{0} \hat{p} N^{2}}{r_{c} \tau_{0}}, \quad b=\tilde{u}_{1 M}\left(r_{c} \pm\right)+\frac{N}{r_{c}} \tilde{w}_{1 M}\left(r_{c} \pm\right),
$$

with $\tilde{\eta}$ a function of $Y$ and $\tilde{\xi}$ given by

$$
\tilde{\eta}=\frac{\tau_{0}}{2 r_{c}}\left(Y-\frac{r_{c} b}{\tau_{0}}\right)^{2}+\tilde{\beta} \cos \tilde{\xi} .
$$

Note here the similarity to the leading-order large-amplitude solution $\mathscr{G}(\hat{\eta})$ found in the weakly nonlinear critical layer of $\S 3$. As in that case, the function $G(\tilde{\eta})$ will be determined at higher order, but to match with the inviscid region we require the asymptotic behaviour

$$
G(\tilde{\eta}) \sim \pm \frac{\left(2 \tau_{0} r_{c}\right)^{1 / 2}}{N} \tilde{\eta}^{1 / 2}+\tilde{w}_{1 M}\left(r_{c} \pm\right) \quad \text { as } \quad \tilde{\eta} \rightarrow \infty,
$$

implied by $(72 c)$ and $(73 c)$. As in our earlier work, the plus sign here refers to the upper part of the critical layer where in this case $Y-r_{c} b / \tau_{0}>\left(2 r_{c} \tilde{\beta}(1-\cos \tilde{\xi}) / \tau_{0}\right)^{1 / 2}$, and the minus sign to the lower part wherein $Y-r_{c} b / \tau_{0}<-\left(2 r_{c} \tilde{\beta}(1-\cos \tilde{\xi}) / \tau_{0}\right)^{1 / 2}$.

In order to determine the phase shift we need to examine the behaviour of higherorder terms. To facilitate this we follow our earlier ideas and define a skewed velocity

$$
\bar{u}_{m}=U_{m}+\frac{N}{r_{c}} W_{m}
$$

for $m=1,3 / 2,2, \ldots$ The equations governing the behaviour of higher-order terms may then be expressed in the form

$$
\begin{gathered}
\bar{u}_{m \tilde{\xi}}+V_{m Y}=\mathscr{F}_{m}^{(1)}, \\
\bar{u}_{1} \bar{u}_{m \tilde{\xi}}+V_{1} \bar{u}_{m Y}+V_{m} \bar{u}_{1 Y}+\left(N / r_{c}\right)^{2} P_{m \tilde{\xi}}=\mathscr{F}_{m}^{(2)}, \\
P_{m Y}=\mathscr{F}_{m}^{(3)}, \\
U_{1} W_{m \tilde{\xi}}+U_{m} W_{1 \tilde{\xi}}+V_{1} W_{m Y}+V_{m} W_{1 Y}+\frac{N}{r_{c}}\left(W_{1} W_{m \tilde{\xi}}+W_{m} W_{1 \tilde{\xi}}\right)+\frac{N}{r_{c}} P_{m \tilde{\xi}}=\mathscr{F}_{m}^{(4)} .
\end{gathered}
$$

The quantities $\mathscr{F}_{m}^{(n)}(m=1,3 / 2,2, \ldots ; n=1,2,3,4)$ are forcing terms arising from the RNS equations which can be written down for any particular $m$ and $n$. If we differentiate $(76 b)$ with respect to $Y$ and use the leading-order solutions (73) we can obtain an equation governing the behaviour of the shear $\bar{u}_{m Y}$. This takes the form

$$
\mp\left(\frac{2 \tau_{0}}{r_{c}}\right)^{1 / 2}(\tilde{\eta}-\tilde{\beta} \cos \tilde{\xi})^{1 / 2} \frac{\partial \bar{u}_{m Y}}{\partial \hat{\xi}}=\frac{\partial \mathscr{F}_{m}^{(2)}}{\partial Y}-\frac{N^{2}}{r_{c}^{2}} \frac{\partial \mathscr{F}_{m}^{(3)}}{\partial \tilde{\xi}}+\frac{\tau_{0}}{r_{c}} \mathscr{F}_{m}^{(1)} .
$$

Here the transformation of $(\tilde{\xi}, Y)$ to characteristic variables $(\hat{\xi}, \tilde{\eta})$, with $\tilde{\xi}=\hat{\xi}$ has 
been performed so that

$$
\frac{\partial}{\partial \tilde{\xi}}=\frac{\partial}{\partial \hat{\xi}}-\tilde{\beta} \sin \tilde{\xi} \frac{\partial}{\partial \tilde{\eta}}, \quad \frac{\partial}{\partial Y}= \pm\left(\frac{2 \tau_{0}}{r_{c}}\right)^{1 / 2}(\tilde{\eta}-\tilde{\beta} \cos \tilde{\xi})^{1 / 2} \frac{\partial}{\partial \tilde{\eta}} .
$$

A similar equation for $W_{m}$ can be obtained and takes the form

$$
\begin{aligned}
\mp\left(\frac{2 \tau_{0}}{r_{c}}\right)^{1 / 2} & (\tilde{\eta}-\tilde{\beta} \cos \tilde{\xi})^{1 / 2} \frac{\partial W_{m}}{\partial \hat{\xi}}=\tilde{\beta} \sin \tilde{\xi} G^{\prime}(\tilde{\eta}) \bar{u}_{m} \\
& +V_{m}\left(\frac{\tau_{0}}{N} \mp\left(\frac{2 \tau_{0}}{r_{c}}\right)^{1 / 2}(\tilde{\eta}-\tilde{\beta} \cos \tilde{\xi})^{1 / 2} G^{\prime}(\tilde{\eta})\right)+\mathscr{F}_{m}^{(4)}-\frac{N}{r_{c}} P_{m \tilde{\xi}}
\end{aligned}
$$

This equation enables $W_{m}$ to be determined once the shear term $\bar{u}_{m Y}$ is found from (77). The corresponding equations to (77) and (78) in SB are their (3.10a) and (3.10b).

In order to induce a phase shift across the critical layer we require the solution for $\left(\bar{u}_{m}, W_{m}, P_{m}\right)$ to possess an odd part about $\tilde{\xi}=\pi$, with $V_{m}$ having an even part. The task here is to find the value of $m$ at which this first occurs. Although our earlier studies suggest this will not occur until $m=5$ it is still vital to consider the smaller values of $m$ first. As in SB the $m=3 / 2$ solution does not give a phase shift and will not be considered further here. Next we consider the $m=2$ stage for which the forcing terms take the form

$$
\begin{gathered}
\mathscr{F}_{2}^{(1)}=-\frac{V_{1}}{r_{c}}+\frac{N Y W_{1 \tilde{\xi}}}{r_{c}^{2}}, \\
\mathscr{F}_{2}^{(2)}=\frac{N Y W_{1} U_{1 \tilde{\xi}}}{r_{c}^{2}}+\frac{N}{r_{c}}\left(\frac{N Y W_{1} W_{1 \tilde{\xi}}}{r_{c}^{2}}-\frac{V_{1} W_{1}}{r_{c}}+\frac{N Y P_{1 \tilde{\xi}}}{r_{c}^{2}}\right), \\
\mathscr{F}_{2}^{(3)}=\frac{W_{1}^{2}}{r_{c}}, \quad \mathscr{F}_{2}^{(4)}=\frac{N Y W_{1} W_{1 \tilde{\xi}}}{r_{c}^{2}}-\frac{V_{1} W_{1}}{r_{c}}+\frac{N Y P_{1 \tilde{\xi}}}{r_{c}^{2}} .
\end{gathered}
$$

Here the controlling equations are (76) with $m=2$ subject to the following matching conditions with the inviscid regions as $Y \rightarrow \pm \infty$ :

$$
\begin{gathered}
U_{2} \sim \frac{\tau_{1}}{r_{c}^{2}} Y^{2}+Y \tilde{u}_{1 M}^{\prime}\left(r_{c} \pm\right)+\tilde{u}_{2 M}\left(r_{c} \pm\right) \\
\quad+\frac{N^{2} A_{0} \hat{p}}{\tau_{0} r_{c}^{2}}\left(-\left(1-\frac{2 \tau_{1}}{\tau_{0}}\right) \ln \left|\frac{Y}{r_{c}}\right|-\frac{1}{3}\left(1-\frac{2 \tau_{1}}{\tau_{0}}\right)-\frac{3 j_{3}}{N^{2}}\right) \cos \tilde{\xi}, \\
V_{2} \sim-\frac{A_{0} \hat{p} N^{2}}{\tau_{0} r_{c}}\left(\left(1-\frac{2 \tau_{1}}{\tau_{0}}\right) \frac{Y}{r_{c}} \ln \left|\frac{Y}{r_{c}}\right|+\left(\frac{1}{3}+\frac{3 j_{3}}{N^{2}}+\frac{1}{3} \frac{\tau_{1}}{\tau_{0}}\right) \frac{Y}{r_{c}}\right) \sin \tilde{\xi}, \\
W_{2} \sim Y \tilde{w}_{1 M}^{\prime}\left(r_{c} \pm\right)-\frac{\hat{p} N A_{0}}{r_{c} \tau_{0}}\left(1-\frac{\tau_{1}}{\tau_{0}}\right) \cos \tilde{\xi}+\tilde{w}_{2 M}\left(r_{c} \pm\right),
\end{gathered}
$$

implied by the asymptotic behaviour (67). In this case equation (77) for the shear $\bar{u}_{2 Y}$ with forcing terms given by (79) can be integrated with respect to $\hat{\xi}$ to yield

$$
\begin{aligned}
-2 r_{c}^{1 / 2} \bar{u}_{2 Y}=\kappa(\tilde{\eta}) \pm & (\tilde{\eta}-\tilde{\beta} \cos \tilde{\xi})^{1 / 2}\left(-\frac{2}{r_{c}}\left(2 \tau_{0}\right)^{1 / 2}\right. \\
& \left.+\frac{8 N^{2}}{r_{c}^{2}} \frac{G G^{\prime}}{\left(2 \tau_{0}\right)^{1 / 2}}-\frac{2 N b}{r_{c}}\left(\frac{2}{\tau_{0}}\right)^{1 / 2} G^{\prime}\right)+\frac{4 N \tilde{\beta} G^{\prime}}{r_{c}^{3 / 2}} \cos \tilde{\xi},
\end{aligned}
$$


with the function $\kappa(\tilde{\eta})$ as yet unknown. From the boundary conditions (80) we deduce

$$
\bar{u}_{2 Y} \sim \frac{2 \tau_{1}}{r_{c}^{2}} Y+\tilde{\lambda}^{ \pm} \quad \text { as } \quad Y \rightarrow \pm \infty,
$$

with $\tilde{\lambda}^{ \pm}=\tilde{u}_{1 M}^{\prime}\left(r_{c} \pm\right)+\left(N / r_{c}\right) \tilde{w}_{1 M}^{\prime}\left(r_{c} \pm\right)$ being constants in the upper and lower regions of the critical layer. The quantity $\tilde{\lambda}^{+}-\tilde{\lambda}^{-}$is the equivalent of the vorticity jump $\hat{\lambda}^{+}-\hat{\lambda}^{-}$found in the weakly nonlinear critical layer of $\S 3$. Applying the boundary condition (82) and the asymptotic form (74) for $G$ to (81) we find that a requirement of the shear term $\kappa(\tilde{\eta})$ is

$$
\kappa(\tilde{\eta}) \sim \mp \frac{2^{3 / 2}}{r_{c} \tau_{0}^{1 / 2}}\left(2 \tau_{1}+\tau_{0}\right) \tilde{\eta}^{1 / 2} \quad \text { as } \quad \tilde{\eta} \rightarrow \infty .
$$

It is worth noting here that for fully developed HPF we have $2 \tau_{1}+\tau_{0}=0$, so that SB's analysis requires $\kappa \rightarrow 0$ as $\tilde{\eta} \rightarrow \infty$.

Provided the forcing terms $\mathscr{F}_{m}^{(1)}, \mathscr{F}_{m}^{(2)}, \mathscr{F}_{m}^{(3)}, \mathscr{F}_{m}^{(4)}$ remain odd, odd, even, odd respectively about $\tilde{\xi}=\pi$, there will be no phase shift. This pattern will continue until viscous terms begin to appear in the forcing. As in SB this occurs at the $m=4$ stage and although it turns out that there is no overall phase shift at this level we are able to determine the function $G(\tilde{\eta})$ there which then completely fixes the $m=1$ solution.

Using ' $O$ ' or ' $E$ ' to represent contributions that are odd or even about $\tilde{\xi}=\pi$, the forcing terms at the $m=4$ stage may be written in the simple form

$$
\begin{gathered}
\mathscr{F}_{4}^{(1)}={ }^{\prime} O ', \quad \mathscr{F}_{4}^{(2)}=U_{1 Y Y}+\frac{N}{r_{c}} W_{1 Y Y}+{ }^{\prime} O ', \\
\mathscr{F}_{4}^{(3)}={ }^{\prime} E, \quad \mathscr{F}_{4}^{(4)}=W_{1 Y Y}+{ }^{\prime} O ',
\end{gathered}
$$

and the shear equation (77) takes the form

$$
\begin{aligned}
& \mp\left(\frac{2 \tau_{0}}{r_{c}}\right)^{1 / 2}(\tilde{\eta}-\tilde{\beta} \cos \tilde{\xi})^{1 / 2} \frac{\partial \bar{u}_{4 Y}}{\partial \hat{\xi}}=\frac{\partial}{\partial Y}\left(U_{1 Y Y}+\frac{N}{r_{c}} W_{1 Y Y}+{ }^{\prime} O^{\prime}\right)-\frac{N^{2}}{r_{c}^{2}} \frac{\partial}{\partial \tilde{\xi}}\left({ }^{\prime} E^{\prime}\right)+{ }^{\prime} O \text { ' } \\
& =\bar{u}_{1 Y Y Y}+{ }^{\circ} O{ }^{\prime}={ }^{\prime} O \text { ', }
\end{aligned}
$$

since $\bar{u}_{1 Y Y Y}$ is zero from (73) and (75). Therefore we see that $\bar{u}_{4 Y}$ is ' $E$ ' and hence $V_{4}$ is ' $O$ ' from (76a) and $P_{4}$ is ' $E$ ' from (76c) and $(84 a, d c)$. After some manipulation the equation governing $W_{4}$ reduces to

$$
\mp \frac{\partial W_{4}}{\partial \hat{\xi}}=\left(\frac{2 \tau_{0}}{r_{c}}\right)^{1 / 2} \frac{\partial}{\partial \tilde{\eta}}\left((\tilde{\eta}-\tilde{\beta} \cos \tilde{\xi})^{1 / 2} G^{\prime}(\tilde{\eta})\right)+{ }^{\prime} O ',
$$

which may be compared with the weakly nonlinear result (51). Thus upon integration we find the solution

$$
\mp W_{4}=\left(\frac{2 \tau_{0}}{r_{c}}\right)^{1 / 2} \frac{\partial}{\partial \tilde{\eta}}\left(G^{\prime}(\tilde{\eta}) \int_{0}^{\hat{\xi}}(\tilde{\eta}-\tilde{\beta} \cos q)^{1 / 2} \mathrm{~d} q\right)+C_{4}(\tilde{\eta})+{ }^{'} E,
$$

with $C_{4}(\tilde{\eta})$ an arbitrary function. We are now in a position to determine the function $G(\tilde{\eta})$ by imposing that $W_{4}$ has periodicity of $2 N \pi$ in $\tilde{\xi}$, i.e. $2 \pi$ in $\theta$. Thus

$$
G^{\prime}(\tilde{\eta})=\frac{\tilde{D}^{ \pm}}{I(\tilde{\eta})}, \quad \text { with } \quad I(\tilde{\eta})=\int_{0}^{2 N \pi}(\tilde{\eta}-\tilde{\beta} \cos q)^{1 / 2} \mathrm{~d} q,
$$

cf. equation (52). The constants $\tilde{D}^{ \pm}$can be determined from the asymptotic form (74) 
as

$$
\tilde{D}^{ \pm}= \pm\left(2 \tau_{0} r_{c}\right)^{1 / 2} \pi
$$

by letting $\tilde{\eta} \rightarrow \infty$ in (85) and using $I \sim 2 N \pi \tilde{\eta}^{1 / 2}$ as $\tilde{\eta} \rightarrow \infty$. From our expression for $G^{\prime}$ we can show that

$$
G(\tilde{\eta}) \sim G_{0} \pm\left(2 \tau_{0} r_{c}\right)^{1 / 2} \pi\left(\frac{\tilde{\eta}^{1 / 2}}{N \pi}+\tilde{J}\right) \quad \text { as } \quad \tilde{\eta} \rightarrow \infty,
$$

with

$$
G(\tilde{\beta})=G_{0}, \quad \tilde{J}=\frac{(2 \tilde{\beta})^{1 / 2}}{8 N \pi} C^{(1)},
$$

where we have uniform vorticity $G_{0}$ within the cat's eye as in the weakly nonlinear critical layer of $\S 3$ and $C^{(1)} \simeq-5.516$ is the same constant that we encountered there. Hence the finite part of the jump in $G(\tilde{\eta})$ across the critical layer is determined as

$$
[[G(\tilde{\eta})]]_{-\infty}^{+\infty}=2\left(2 \tau_{0} r_{c}\right)^{1 / 2} \pi \tilde{J} .
$$

This result will be useful when we turn to calculating the phase shift presently.

Although both $W_{4}$ and $U_{4}$ have non-zero odd parts, these tend to zero as $Y \rightarrow \pm \infty$, yielding no overall phase shift. The final stage we need to consider (as anticipated earlier) is $m=5$ where the shear equation takes the form

$$
\begin{aligned}
\mp\left(\frac{2 \tau_{0}}{r_{c}}\right)^{1 / 2} & (\tilde{\eta}-\tilde{\beta} \cos \tilde{\xi})^{1 / 2} \frac{\partial \bar{u}_{5 Y}}{\partial \hat{\xi}} \\
& =\bar{u}_{2 Y Y Y}-\frac{N}{r_{c}^{2}} V_{1} W_{4 Y}+\frac{N \tau_{0}}{r_{c}^{3}} Y W_{4 \tilde{\xi}}-\frac{2 N^{2}}{r_{c}^{3}} \frac{\partial}{\partial \tilde{\xi}}\left(W_{1} W_{4}\right)+{ }^{\prime} O ' .
\end{aligned}
$$

By substituting for $\bar{u}_{2 Y}$ from (81), integrating with respect to $\hat{\xi}$ and demanding periodicity, we can derive an equation governing the behaviour of the shear term $\kappa(\tilde{\eta})$ first introduced in (81). Substituting for $G^{\prime}$ from (85) and integrating with respect to $\tilde{\eta}$ we finally obtain

$$
\frac{\tau_{0} r_{c}}{4 N^{2}} \kappa^{\prime}(\tilde{\eta})=\mp \frac{2 \pi N}{r_{c}} \frac{\left(2 \tau_{0}\right)^{1 / 2}}{\tilde{D}^{ \pm}} \tilde{\eta}\left(G^{\prime}(\tilde{\eta})\right)^{3}-\frac{\tau_{0}}{N r_{c}^{1 / 2}} \tilde{\eta} G^{\prime \prime}(\tilde{\eta})-\frac{D_{2}^{ \pm}}{\tilde{D}^{ \pm}} G^{\prime}(\tilde{\eta})
$$

which reduces to SB's equation (3.20) in the case of HPF. Use of the asymptotic condition (87) for $G^{\prime}$ and (86) for $\tilde{D}^{ \pm}$implies that

$$
\frac{\tau_{0} r_{c}}{4 N^{2}} \kappa^{\prime} \sim\left(\mp\left(2 \tau_{0}\right)^{1 / 2} \frac{\tau_{0}}{4 N^{2}}-\frac{D_{2}^{ \pm}}{2 N \pi}\right) \tilde{\eta}^{-1 / 2} \text { as } \tilde{\eta} \rightarrow \infty,
$$

thus fixing the values of the constants $D_{2}^{ \pm}$as

$$
D_{2}^{ \pm}= \pm\left(2 \tau_{0}\right)^{1 / 2} \frac{\pi}{N} \tau_{1}
$$

from comparison of (83) with (92). Our expression for $\kappa^{\prime}$ can now be written as

$$
\frac{\tau_{0} r_{c}}{4 N^{2}} \kappa^{\prime}(\tilde{\eta})=-\frac{2 N}{r_{c}^{3 / 2}} \tilde{\eta} G^{3}-\frac{\tau_{0}}{N r_{c}^{1 / 2}} \tilde{\eta} G^{\prime \prime}-\frac{\tau_{1}}{N r_{c}^{1 / 2}} G^{\prime} .
$$

Integrating this equation and applying the uniform vorticity condition $\kappa=\kappa_{0}$ when 
$\tilde{\eta}=\tilde{\beta}$ we obtain

$$
\begin{aligned}
\frac{\tau_{0} r_{c}}{4 N^{2}}\left(\kappa(\tilde{\eta})-\kappa_{0}\right)=-\frac{\tau_{0}}{N r_{c}^{1 / 2}}\left(\tilde{\eta} G^{\prime}(\tilde{\eta})-G(\tilde{\eta})-\tilde{\beta} G^{\prime}(\tilde{\beta})+G_{0}\right) & \\
& -\frac{\tau_{1}}{N r_{c}^{1 / 2}}\left(G(\tilde{\eta})-G_{0}\right)-\frac{2 N}{r_{c}^{3 / 2}} \int_{\tilde{\beta}}^{\tilde{\eta}} q\left(G^{\prime}(q)\right)^{3} \mathrm{~d} q .
\end{aligned}
$$

Letting $\tilde{\eta} \rightarrow \infty$, and using the asymptotic form (87) for $G$ we find

$$
\begin{aligned}
\frac{\tau_{0} r_{c}}{4 N^{2}}\left(\kappa(\tilde{\eta})-\kappa_{0}\right) \sim \mp \frac{\left(2 \tau_{0}\right)^{1 / 2}}{2 N^{2}} \tilde{\eta}^{1 / 2}\left(\tau_{0}+2 \tau_{1}\right) \pm\left(2 \tau_{0}\right)^{1 / 2} \frac{\pi}{N}\left(\tau_{0}-\tau_{1}\right) \tilde{J} \\
\pm \frac{\tau_{0}^{3 / 2} \tilde{\beta}^{1 / 2} \pi}{4 N^{2}} \mp \frac{\left(2 \tau_{0}\right)^{3 / 2}}{4 N^{2}} \tilde{\beta}^{1 / 2}\left(C^{(2)}-2\right) .
\end{aligned}
$$

Here, the term proportional to $\tilde{\eta}^{1 / 2}$ matches with the earlier requirement of (83) while the final term on the right-hand side arises from the integral in (94), where

$$
C^{(2)}=\frac{8 \pi^{3} N^{3}}{\tilde{\beta}^{1 / 2}} \int_{\tilde{\beta}}^{\infty}\left(\frac{s}{I^{3}}-\frac{1}{8 \pi^{3} N^{3} s^{1 / 2}}\right) \mathrm{d} s \simeq 0.1564,
$$

from numerical computation. This final term appears to have been neglected in SB's analysis. The finite part of the jump in $\kappa(\tilde{\eta})$ across the critical layer is therefore given by

$$
\frac{\tau_{0} r_{c}}{4 N^{2}}[[\kappa(\tilde{\eta})]]_{-\infty}^{+\infty}=2\left(2 \tau_{0}\right)^{1 / 2} \frac{\pi}{N}\left(\tau_{0}-\tau_{1}\right) \tilde{J}+\frac{\tau_{0}^{3 / 2}}{2 N^{2}} \tilde{\beta}^{1 / 2} \pi-\frac{\left(2 \tau_{0}\right)^{3 / 2}}{2 N^{2}}\left(C^{(2)}-2\right) \tilde{\beta}^{1 / 2} .
$$

Hence, using (81) and the expression (88) for $\widetilde{J}$, we determine the vorticity jump across the critical layer as

$$
\begin{aligned}
\tilde{\lambda}^{+}-\tilde{\lambda}^{-} & =\left[\left[\bar{u}_{2 Y}\right]\right]_{-\infty}^{+\infty}=-\frac{1}{2 r_{c}^{1 / 2}}[[\kappa(\tilde{\eta})]]_{-\infty}^{+\infty} \\
& =-\frac{\tau_{0}^{1 / 2} \tilde{\beta}^{1 / 2}}{r_{c}^{3 / 2}}\left(\left(2-\frac{\tau_{1}}{\tau_{0}}\right) C^{(1)}+\pi+2^{3 / 2}\left(2-C^{(2)}\right)\right) .
\end{aligned}
$$

We are now finally in a position to determine the phase shift explicitly. If we write

$$
\left[\left[\bar{u}_{5}\right]\right]_{-\infty}^{+\infty}=\sum_{n=1}^{\infty}\left(\beta_{n} \sin n \tilde{\xi}+\gamma_{n} \cos n \tilde{\xi}\right),
$$

then the coefficient of relevance to the phase shift is $\beta_{1}(=\tilde{\phi}$ say) given by

$$
\tilde{\phi}=\frac{1}{N \pi} \int_{0}^{2 N \pi}\left[\left[\bar{u}_{5}\right]\right]_{-\infty}^{+\infty} \sin \tilde{\xi} \mathrm{d} \tilde{\xi}=\frac{1}{N \pi} f_{-\infty}^{\infty}\left(\int_{0}^{2 N \pi} \bar{u}_{5 Y} \sin \tilde{\xi} \mathrm{d} \tilde{\xi}\right) \mathrm{d} Y .
$$

By using our expression for $\bar{u}_{5 Y}$ (deduced from (90)) and integrating we eventually find

$$
\tilde{\beta} \tilde{\phi}=-2\left(\tilde{\lambda}^{+}-\tilde{\lambda}^{-}\right)+\frac{4}{r_{c}^{2}}\left(2 \tau_{0} r_{c}\right)^{1 / 2} N \pi \tilde{J},
$$

using an identical approach to that explained in an appendix to SB. This is the strongly nonlinear equivalent of the result (48). Substitution of (88) and (95) into (96) 
leaves the phase shift satisfying

$$
\tilde{\beta} \tilde{\phi}=\left(\frac{\tau_{0} \tilde{\beta}}{r_{c}^{3}}\right)^{1 / 2}\left(\left(5-\frac{2 \tau_{1}}{\tau_{0}}\right) C^{(1)}+2 \pi+2^{5 / 2}\left(2-C^{(2)}\right)\right) .
$$

Now, using the asymptotic forms for $U_{2}, W_{2}$ in (80) we deduce that, since the coefficient of the logarithmic term in the asymptotic expression for $\bar{u}_{2}$ is $-N^{2} A_{0} \hat{p}\left(1-2 \tau_{1} / \tau_{0}\right) / r_{c}^{2} \tau_{0}$, we must have the following relation between $\tilde{\phi}$ and $\Phi$ :

$$
\tilde{\phi}=\frac{A_{0} \hat{p} N^{2}}{r_{c}^{2} \tau_{0}}\left(1-\frac{2 \tau_{1}}{\tau_{0}}\right) \Phi .
$$

Substituting this relation into (97) we find the following expression for the scaled phase shift $\Phi$ as a function of disturbance amplitude $A_{0}$ :

$$
\Phi=\frac{\tau_{0}^{2} r_{c}}{A_{0}^{3 / 2} \hat{p}^{3 / 2} N^{3}\left(1-\frac{2 \tau_{1}}{\tau_{0}}\right)}\left(\left(5-\frac{2 \tau_{1}}{\tau_{0}}\right) C^{(1)}+2 \pi+2^{5 / 2}\left(2-C^{(2)}\right)\right) .
$$

Expression (98) should be compared with equation (3.25) of SB with allowance made for the final term involving $C^{(2)}$ which was missed in their analysis. This phase shift is exactly counterbalanced by that produced by the viscous wall layer in a similar way to the upper branch linear stability problem, and this balancing will determine the threshold amplitude $A_{0}$ for the nonlinear instability mechanism to occur.

\subsection{The viscous wall layer and the balancing of phase shifts}

The dynamics of the wall layer, which is of $O\left(\tilde{\alpha}^{-1 / 2}\right)$ thickness, are very similar to those of region IV in the linear analysis of $\S 2$ (although the current disturbance is larger), so the details are not presented here. Instead we simply quote the result we require, namely that

$$
\tilde{v} \sim-\tilde{\alpha}^{2 / 3} \frac{N^{2} A_{0}}{c_{0}}(1-r) \sin \tilde{\xi}+\tilde{\alpha}^{1 / 6} \frac{N^{2} A_{0}}{2^{1 / 2} c_{0}^{3 / 2}}(\cos \tilde{\xi}+\sin \tilde{\xi}) \quad \text { as } \quad r \rightarrow 1,
$$

which should be compared with the linear result (29). The first term here matches with the behaviour of $G_{2}$ in the inviscid region, while the second term implies that

$$
\tilde{v}_{5} \rightarrow \frac{N^{2} A_{0}}{2^{1 / 2} c_{0}^{3 / 2}}(\cos \tilde{\xi}+\sin \tilde{\xi}) \quad \text { as } \quad r \rightarrow 1,
$$

from comparison of (99) with (61). Thus we see from (99) that the phase shift induced in the normal velocity disturbance by the wall layer is in tune with that induced across the critical layer. Now that we have expression (100) we can determine the disturbance amplitude and its dependence upon $\tilde{t}$ precisely.

\subsection{Determination of the nonlinear disturbance amplitude}

Our critical layer analysis has revealed that the components $\left(\tilde{u}_{5}, \tilde{v}_{5}, \tilde{w}_{5}, \tilde{p}_{5}\right)$ contain terms of the form

$$
A_{0}\left(F_{5}(r) \sin \tilde{\xi}, G_{5}(r) \cos \tilde{\xi}, H_{5}(r) \sin \tilde{\xi}, Q_{5}(r) \sin \tilde{\xi}\right)
$$


where $G_{5}(1)=N^{2} /\left(2^{1 / 2} c_{0}^{3 / 2}\right)$ from (100). After substitution into the RNS equations and some manipulation we find that $Q_{5}$ satisfies the same equation as $Q_{2}$, namely

$$
\left(\tilde{u}_{0}-c_{0}\right)\left(Q_{5}^{\prime \prime}+\frac{1}{r} Q_{5}^{\prime}-\frac{N^{2}}{r^{2}} Q_{5}\right)=2 \tilde{u}_{0}^{\prime} Q_{5}^{\prime},
$$

subject to

$$
Q_{5}(0)=0, \quad Q_{5}^{\prime}(1)=-\frac{N^{2}}{2^{1 / 2} c_{0}^{1 / 2}},
$$

and the jump condition

$$
\left[\left[Q_{5}\right]\right]_{-}^{+}=-\frac{N^{2} \hat{p}}{3}\left(1-\frac{2 \tau_{1}}{\tau_{0}}\right)\left(\frac{r_{c}-r}{r_{c}}\right)^{3} \Phi,
$$

deduced from the pressure behaviour in (67d) and the jump (69). Consideration of the Wronskian of $Q_{2}$ and $Q_{5}$ and the boundary conditions (101) leads us to the result

$$
\frac{r}{\left(r_{c}^{2}-r^{2}\right)^{2}}\left(Q_{2} Q_{5}^{\prime}-Q_{5} Q_{2}^{\prime}\right)= \begin{cases}-N^{2} /\left(2^{1 / 2} c_{0}^{5 / 2}\right), & r>r_{c} \\ 0, & r<r_{c} .\end{cases}
$$

Thus, upon applying the jump condition (102) we obtain

$$
\Phi=-\frac{\tau_{0}^{3}}{\hat{p}^{2} 2^{1 / 2} c_{0}^{5 / 2}\left(\tau_{0}-2 \tau_{1}\right)},
$$

giving the phase shift in terms of the quantities $\hat{p}$ and $c_{0}$ which can be calculated numerically. Equating our two expressions (98) and (103) for $\Phi$ we obtain

$$
A_{0}=\frac{2^{1 / 3} r_{c}^{2 / 3} \hat{p}^{1 / 3} c_{0}^{5 / 3}}{N^{2}}\left(\left(5-\frac{2 \tau_{1}}{\tau_{0}}\right)\left(-C^{(1)}\right)-2 \pi-2^{5 / 2}\left(2-C^{(2)}\right)\right)^{2 / 3},
$$

where all the quantities on the right-hand side are either known analytically or can be easily computed numerically. Since the basic flow is time-dependent, these quantities all depend on $\tilde{t}$ and so (104) gives the development of $A_{0}$ with time. This result should be compared with the corrected form of SB's equation (3.26) for the special case of HPF. The asymptotic structure of the nonlinear neutral modes is shown in figure 3 where $\tilde{t}$ is $O(1)$.

To determine $c_{0}$ and $\hat{p}$ we need to solve the reduced Rayleigh equation (65) numerically. This is carried out in the next section together with the explicit determination of the amplitude of the neutral modes.

\section{Numerical solution of the reduced Rayleigh equation}

The numerical problem under study here is the solution of

$$
\left(\tilde{u}_{0}-c_{0}\right)\left(Q_{2}^{\prime \prime}+\frac{1}{r} Q_{2}^{\prime}-\frac{N^{2}}{r^{2}} Q_{2}\right)=2 \tilde{u}_{0}^{\prime} Q_{2}^{\prime} \quad(0 \leqslant r \leqslant 1,0 \leqslant \tilde{t}<\infty),
$$

where $\tilde{u}_{0}$ is given by (60) and depends on $r$ and $\tilde{t}$ with primes here representing differentiation with respect to $r$. The boundary conditions are

$$
Q_{2}(0)=0, \quad Q_{2}^{\prime}(1)=0 .
$$

In addition we need to apply the condition of zero phase shift at $r=r_{c}$ where $\tilde{u}_{0}\left(r_{c}\right)=c_{0}$. From the asymptotic expansion (67d) of $Q_{2}$ as $r \rightarrow r_{c}-$ we can derive the 


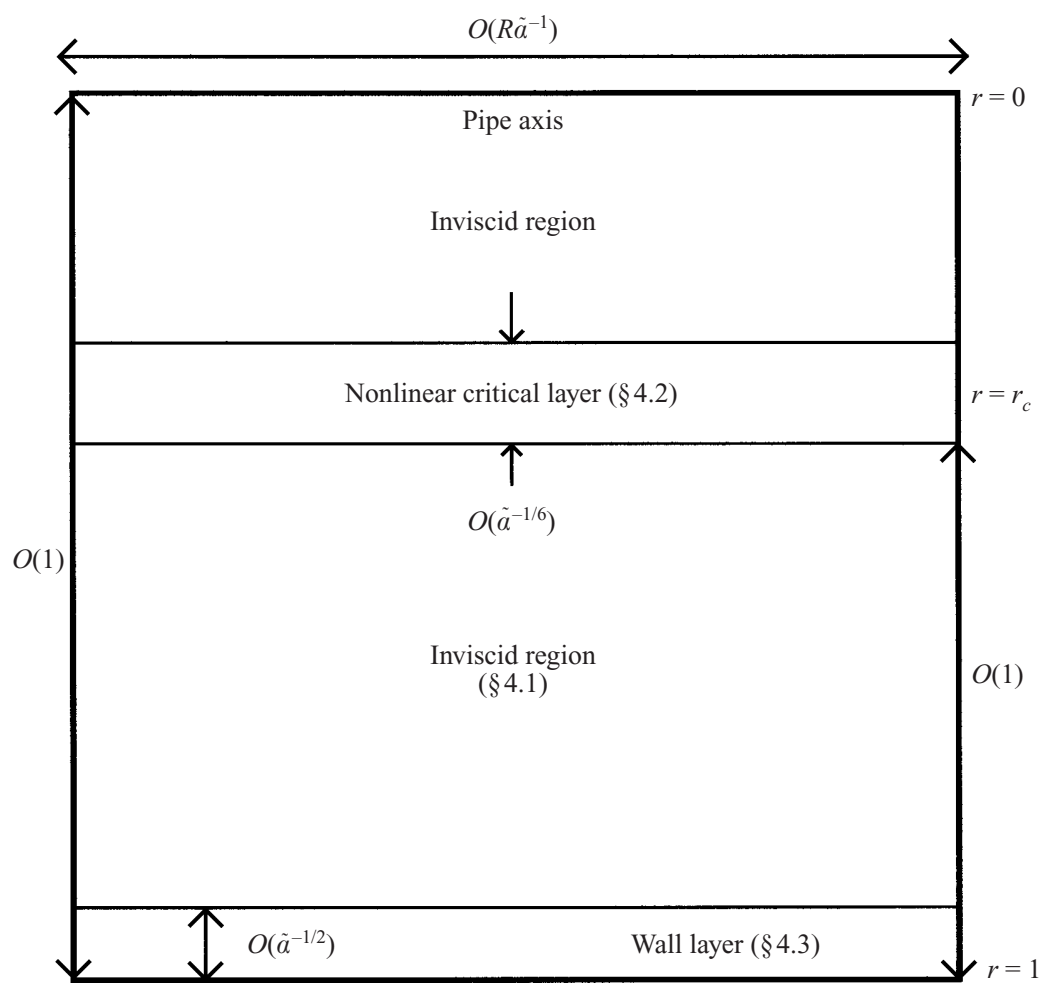

FIGURE 3. The nonlinear neutral mode structure of $\S 4$ for $t=R \tilde{t}$ with $\tilde{t}$ of $O(1)$ and $\tilde{\alpha} \gg 1$.

jump in $Q_{2}^{\prime} / Q_{2}$ as

$$
\left[\frac{Q_{2}^{\prime}}{Q_{2}}\right]_{-}^{+} \sim-\frac{2 N^{2}}{r_{c}}|\tilde{\varepsilon}|+\frac{2 N^{2}}{r_{c}}\left(1-4\left(\frac{\tau_{1}}{\tau_{0}}\right)^{2}\right)|\tilde{\varepsilon}|^{3} \ln |\tilde{\varepsilon}|,
$$

where $\tilde{\varepsilon}=\left(r_{c}-r\right) / r_{c}$.

Our numerical approach is as follows. First for given values of time $\tilde{t}$ and azimuthal wavenumber $N$ we guess a value for the critical layer location $r_{c}$ in the range $0<r_{c}<1$. Then near the regular singular point at $r=0$ we expand $Q_{2}$ in a power series and calculate the quantities $Q_{2}\left(r_{0}\right), Q_{2}^{\prime}\left(r_{0}\right)$ where $r_{0}$ is small. These values are used to initiate a Runge-Kutta scheme which advances the solution as far as $r=r_{c}(1-\tilde{\varepsilon})$ with $\tilde{\varepsilon}$ suitably small. Next, the jump condition (106) allows us to calculate $Q_{2}$ and its derivative at $r=r_{c}(1+\tilde{\varepsilon})$. Finally, the Runge-Kutta scheme is employed a second time to compute the solution for $r_{c}(1+\tilde{\varepsilon})<r \leqslant 1$. In particular we obtain a value for $Q_{2}^{\prime}(1)$. Newton iteration on $r_{c}$ is then applied until $Q_{2}^{\prime}(1)=0$ to some acceptable tolerance. Once the value of $r_{c}$ is found, the value of $Q_{2}(1)$ is known. Our theory normalizes this value to unity so the value of $\hat{p}$ follows from $\hat{p}=Q_{2}\left(r_{c}\right) / Q_{2}(1)$. The wave speed $c_{0}$ can then be found from the relation $\tilde{u}_{0}\left(r_{c}\right)=c_{0}$ and the amplitude of the neutral mode can be determined from (104). The procedure is repeated for a range of values of $\tilde{t}$ and $N$.

The results for the critical layer location $r_{c}$ versus $\tilde{t}$ are shown for various values of $N$ in figure $4(a, b)$. It appears that modes with $N \geqslant 2$ only exist up to a small value of $\tilde{t}=t_{c}$ say, with $t_{c}$ decreasing as $N$ increases. As $\tilde{t} \rightarrow t_{c}-$ we see that $r_{c} \rightarrow 0$ and 

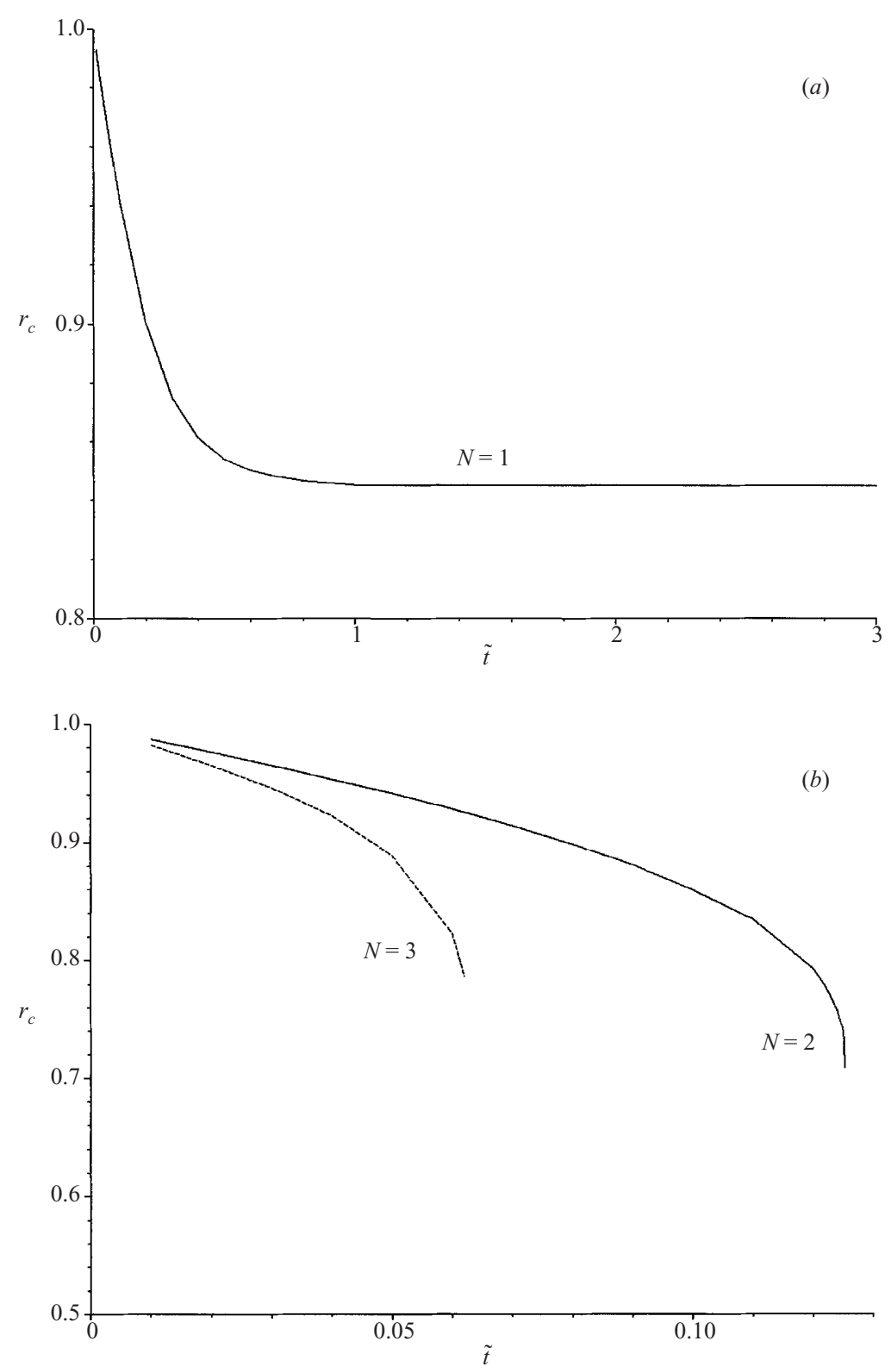

Figure 4. (a) Critical layer location $r_{c}$ versus $\tilde{t}$ for $N=1 ;(b) r_{c}$ versus $\tilde{t}$ for higher azimuthal wavenumbers, $N=2$ and $N=3$.

the critical layer therefore moves towards the pipe axis. The behaviour for $N=1$ however, is quite different. In this case there is no cut-off in $\tilde{t}$, and we see that $r_{c}$ decreases monotonically from unity to a value of about 0.846 as $\tilde{t} \rightarrow \infty$. In figure 5 we show the corresponding behaviour of the wave speed for $N=1$ with $c_{0}$ increasing monotonically to approximately 0.284 as $\tilde{t} \rightarrow \infty$. Figure 6 presents the wave amplitude $A_{0}$ (calculated from (104)) as a function of $\tilde{t}$ for the $N=1$ mode, and we see again monotonic growth from zero amplitude at $\tilde{t}=0$ to a finite value as $\tilde{t} \rightarrow \infty$. 


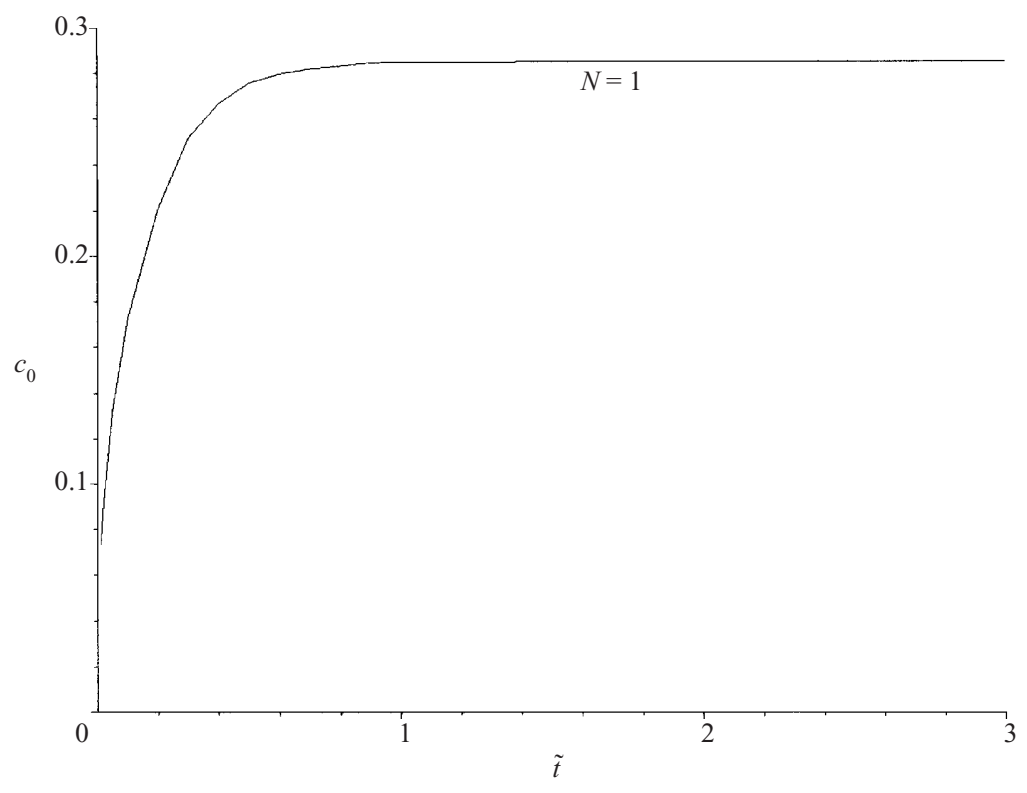

FIGURE 5. Wave speed $c_{0}$ versus $\tilde{t}$ from the results of the computations of $\S 5$ for azimuthal wavenumber $N=1$.

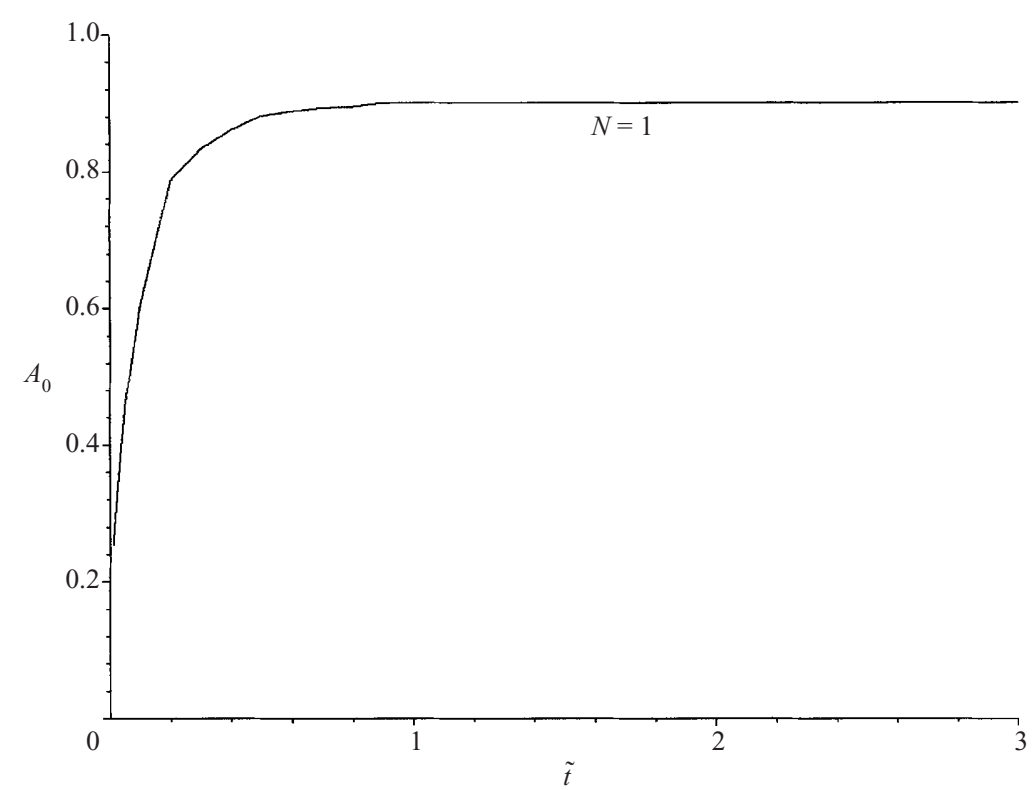

FIGURE 6. Scaled neutral wave amplitude $A_{0}$ as a function of $\tilde{t}$ for $N=1$.

Of particular interest to us here are the limits $\tilde{t} \rightarrow 0, \tilde{t} \rightarrow \infty$ since, as we shall see, the former matches back to the weakly nonlinear disturbance theory of $\S \S 2$ and 3 while the latter limit leads to the fully developed HPF whose stability properties are certainly not fully understood. We examine both limits in some detail in the next section. 


\section{The limiting cases}

\subsection{The limit $\tilde{t} \rightarrow 0$}

When $\tilde{t}$ is small the basic flow acquires the two-tiered form exploited in the linear analysis of $\S 2$. In terms of the current notation we have at leading order

$$
\tilde{u}_{0}= \begin{cases}4 \tilde{t}, & r \sim O(1) \\ 4 \tilde{t} u_{B}(\eta), & r=1-\tilde{t}^{1 / 2} \eta,\end{cases}
$$

with $u_{B}(\eta)$ given in (5). The inviscid region described in $\S 4.1$ splits into three subregions in a very similar way to the linear structure outlined in $\S 2$. Since the analysis is almost identical we present only brief details here.

\subsubsection{The inviscid subregions}

Region I has $r$ of $O(1)$ and the reduced Rayleigh equation simplifies to

$$
Q_{2}^{\prime \prime}+\frac{1}{r} Q_{2}^{\prime}-\frac{N^{2}}{r^{2}} Q_{2}=0,
$$

so that the solution which is regular at $r=0$ is $Q_{2}=r^{N}$, where, as in $\S 2$, we have normalized so that the pressure is unity at the pipe wall.

In region II the basic flow adopts its lower-tier form and we have

$$
r=1-\tilde{t}^{1 / 2} \eta, \quad Q_{2}=1+\tilde{t}^{1 / 2} q_{1}(\eta)+\cdots, \quad \text { with } q_{1}^{\prime}(\eta)=-N\left(u_{B}(\eta)\right)^{2} .
$$

Region III balances the near-wall shear flow with the wave speed and has

$$
\begin{gathered}
u_{B}(\eta)=\tilde{t}^{1 / 2} \lambda_{0} Y+\cdots, \quad r=1-\tilde{t} Y, \\
c_{0}=4 \tilde{t}^{3 / 2} \tilde{c}_{0}+\cdots, \quad Q_{2}=1+\tilde{t}^{2} \hat{Q}_{1}(Y)+\cdots,
\end{gathered}
$$

with $\lambda_{0}=2 / \sqrt{\pi}$. From substitution into the reduced Rayleigh equation (65), we find

$$
\hat{Q}_{1}^{\prime}(Y)=-\frac{N^{2}}{\lambda_{0}}\left(\lambda_{0} Y-\tilde{c}_{0}\right)-N\left(\lambda_{0} Y-\tilde{c}_{0}\right)^{2} .
$$

The inviscid requirement that $\hat{Q}_{1}^{\prime}(0)=0$ leads to the eigenrelation

$$
\frac{N^{2} \tilde{c}_{0}}{\lambda_{0}}-N \tilde{c}_{0}^{2}=0
$$

with solution $\tilde{c}_{0}=N / \lambda_{0}=N \sqrt{\pi} / 2$. We therefore obtain the result

$$
c_{0} \sim \frac{4 N}{\lambda_{0}} \tilde{t}^{3 / 2} \text { as } \tilde{t} \rightarrow 0,
$$

in accordance with the expectation (59b). In addition the asymptotic form (109) agrees well with that obtained from the computations of $\S 5$ as $\tilde{t} \rightarrow 0$. It is also worth noting that since $\tilde{\alpha} c \sim O\left(\tilde{t}^{-3}\right)$ as $\tilde{t} \rightarrow 0$ (from (55) and (109)), derivatives with respect to $\tilde{t}$ can still be neglected and it is therefore valid from a mathematical point of view to apply this limit to the nonlinear problem formulated in $\S 4$.

\subsubsection{The phase shift and amplitude}

Of equal interest to us here is how the amplitude $A_{0}$ and the phase shift $\Phi$ behave in this limit. To find the amplitude dependence we examine (104) in the limit $\tilde{t} \rightarrow 0$. From (108) above we see that $\hat{p} \sim 1$ to leading order since the critical layer is now 
sited close to the wall. We also find that

$$
\tau_{0} \sim \frac{8 \tilde{t}^{1 / 2}}{\pi^{1 / 2}}, \quad \tau_{1} \rightarrow-2 \quad \text { as } \quad \tilde{t} \rightarrow 0,
$$

from (5), (68), (107) and the fact that $r_{c} \rightarrow 1$ as $\tilde{t} \rightarrow 0$. Using this information and the asymptotic form for the wave speed found above we deduce that

$$
A_{0} \sim \frac{2^{13 / 3}}{N^{1 / 3} \lambda_{0}^{7 / 3}}\left(\lambda_{1} C^{(1)}\right)^{2 / 3} \tilde{t}^{13 / 6} \quad \text { as } \quad \tilde{t} \rightarrow 0,
$$

providing a direct match (cf. $(56 a, b a))$ between the weakly nonlinear theory of $\S 3$ and the strongly nonlinear theory presented here. In addition, from examination of (98) in this limit we can obtain the behaviour of the phase shift as

$$
\Phi \sim \frac{\lambda_{0}^{11 / 2}}{(2 N)^{5 / 2} \lambda_{1}} \tilde{t}^{-9 / 4} \text { as } \tilde{t} \rightarrow 0,
$$

in accordance with $(56 a, b b)$, and providing another exact match back to the weakly nonlinear regime.

We can now complete the connection of the weakly nonlinear neutral stability of the developing flow with the nonlinear neutral stability of HPF by analysing the limit of the nonlinear problem of $\S 4$ as $\tilde{t} \rightarrow \infty$.

\subsection{The limit $\tilde{t} \rightarrow \infty$}

From the results of $\S 4$ and the form of the basic flow (60) we have

$$
\tilde{u}_{0} \rightarrow 1-r^{2}, \quad c_{0} \rightarrow c_{01}-, \quad r_{c} \rightarrow r_{c 1}+\quad \text { as } \tilde{t} \rightarrow \infty,
$$

with $\tau_{0} \rightarrow 2 r_{c 1}^{2}, \tau_{1} \rightarrow-r_{c 1}^{2}$. For this to be a valid limit of the nonlinear problem we require $\tilde{\alpha} c \gg 1 / \tilde{t}$ and so we must have $\hat{A} \gg O\left(\tilde{t}^{7 / 6}\right)$ from (55). Given a sufficiently large amplitude $\hat{A}$ then, at large $\tilde{t}$ the reduced Rayleigh equation becomes to leading order

$$
Q_{2}^{\prime \prime}+\left(\frac{1}{r}+\frac{4 r}{r_{c 1}^{2}-r^{2}}\right) Q_{2}^{\prime}-\frac{N^{2}}{r^{2}} Q_{2}=0, \quad Q_{2}(0)=Q_{2}^{\prime}(1)=0,
$$

with zero phase shift across $r=r_{c 1}$. The appropriate solution satisfying the condition at $r=0$ and zero phase shift is

$$
Q_{2} \propto r^{N} \operatorname{Re}\left[\int_{0}^{1} s^{-\mu_{1}}(1-s)^{\mu_{2}}\left(1-\left(\frac{r}{r_{c 1}}\right)^{2} s\right)^{\mu_{3}} \mathrm{~d} s\right],
$$

where the constants $\mu_{i}$ are given by

$$
\mu_{1}=2-\frac{1}{2} N-\frac{1}{2}\left(N^{2}+4\right)^{1 / 2}, \quad \mu_{2}=\mu_{1}+N-1, \quad \mu_{3}=3-\mu_{1}-N .
$$

The value of $r_{c 1}$ can be calculated by applying $Q_{2}^{\prime}(1)=0$ to (110). This gives the unique solution

$$
r_{c 1} \simeq 0.846, \quad c_{01} \simeq 0.284, \quad \hat{p}=Q_{2}\left(r_{c 1}\right) \simeq 1.004 \text { for } \quad N=1
$$

(where we have normalized such that $Q_{2}(1)=1$ ) and it can be proved rigorously that no real solutions exist for any other integer values of $N$ (see Walton 2001 for more details). This result is consistent with our numerical observation that no neutral modes with $N \geqslant 2$ survive beyond a finite value of $\tilde{t}$ in the computations of $\S 5$. The values given in (111) are identical to those found numerically by SB in their investigation of 
the nonlinear stability of HPF and also agree with those found in our computations. Thus we see that as $\tilde{t} \rightarrow \infty$ the nonlinear neutral modes in the impulsively started flow develop into the SB modes (or more accurately the small-wavenumber limit of the SB modes). The amplitude of these neutral modes is given by the large- $\tilde{t}$ limit of (104) with $N=1$ :

$$
A_{01}=2^{1 / 3} r_{c 1}^{2 / 3}\left(Q_{2}\left(r_{c 1}\right)\right)^{2 / 3} c_{01}^{5 / 3}\left(-6 C^{(1)}-2 \pi-2^{5 / 2}\left(2-C^{(2)}\right)\right)^{2 / 3} \simeq 0.894,
$$

while the phase shift across the critical layer is $\tilde{\alpha}^{-1 / 2} \Phi_{1}$, with $\Phi_{1}$ obtained from the limit of (103) as $\tilde{t} \rightarrow \infty$ as

$$
\Phi_{1}=\frac{-2 r_{c 1}^{4}}{\left(Q_{2}\left(r_{c 1}\right)\right)^{2} 2^{1 / 2} c_{01}^{5 / 2}} \simeq-16.71 .
$$

These values agree with the values at large $\tilde{t}$ obtained in the computations of $\S 5$ and complete the connection between the weakly nonlinear stability of the impulsively started flow and the nonlinear stability of Hagen-Poiseuille flow.

\section{Conclusion}

By using asymptotic methods we have followed the evolution of a small neutral disturbance at high Reynolds number from its initial weakly nonlinear stage at the upper branch time, when the basic flow is in an undeveloped state given specifically by (3), all the way to its strongly nonlinear form in the fully developed HPF into which the basic flow evolves as $t \rightarrow \infty$ on the $O(R)$ time scale. The development can be described analytically only for the crucial disturbance size which we have seen is $O\left(R^{-22 / 27}\right)$ at $O\left(R^{7 / 9}\right)$ times after the fluid is set into motion. The amplitude of the neutral wave adjusts over the slow time scale associated with the basic flow, increasing to $O\left(R^{-2} \tilde{\alpha}^{5 / 3}\right)$ as the fully developed state is attained. This agrees with the small-wavenumber limit of Smith \& Bodonyi's work which was mentioned in the introduction.

To explain the results of our analysis and their implications we consider a thought experiment that possibly may be realized in an experimental situation. We confine our attention to $O(R)$ distances from the pipe entrance thus ensuring that the basic developing flow is independent of $x$. A pressure gradient is imposed along the pipe (corresponding to a given large Reynolds number) and the fluid is set into motion. Care is taken at this stage to keep the flow as disturbance free as possible.

Suppose that very soon after the fluid in the pipe has been set into motion, a small spiral-wave disturbance of a fixed (long) wavelength is introduced into the flow. According to linear theory there will be a time interval (bounded by the lower and upper branch times) during which the wave experiences amplification. Provided the disturbance is still sufficiently small when the upper branch time is reached (at which instant the disturbance is neutral) the wave will subsequently decay in time and ultimately the basic flow evolves into HPF.

If however the disturbance has reached the critical size $O\left(R^{-22 / 27}\right)$ at the $O\left(R^{7 / 9}\right)$ upper branch time, the analysis in this paper shows that the wave can remain in neutral, right into the fully developed regime, by adjusting its amplitude and frequency over the slow time scale associated with the mean flow. Ultimately, as $\tilde{t} \rightarrow \infty$ we obtain a small-amplitude long-wavelength neutral wave superimposed on the fully developed HPF.

All of the above assumes that the azimuthal wavenumber of the linear disturbance 
is equal to unity. If this is not the case and we have a combination of azimuthal modes, then the evolution process is not clear-cut since the numerical results of $\S 5$ indicate that a neutral wave with $N \neq 1$ does not persist in a simple form beyond $t=R t_{s}$ say, where $t_{s}$ depends on $N$. The ultimate fate of these disturbances is an area worthy of further study.

Returning to the $N=1$ case, since the structure described here arises from upper branch linear stability properties, we expect that shorter-wavelength disturbances of this amplitude will decay temporally while there will be at least one temporally unstable mode with a longer wavelength than the neutral mode. Further study is called for in order to confirm this and also to investigate the role that the lower branch modes play.

The idea that a nonlinear wave can remain neutral by adjusting its frequency and amplitude over the time scale of the mean flow is reminiscent of vortex-wave interaction theory (Hall \& Smith 1991) in which the evolution of a nonlinear neutral wave is followed, and the effect of the mean flow on the wave occurs as a purely parametric effect.

For the first time then, an unambiguous, natural path leading ultimately to the nonlinear instability of HPF can be clearly seen, although of course a number of questions remain to be addressed. Apart from those mentioned above, the most important next step is to verify the existence of the nonlinear neutral modes numerically either from the solution of the full Navier-Stokes equations at finite $R$, or more tractably, from the solution of the RNS equations (58) at large but finite values of $\tilde{\alpha}$. Although the analysis presented here is only strictly valid at asymptotically large Reynolds number, we believe that by demonstrating that the critical layer moves away from the wall as time increases to $O(1)$ distances at $O(R)$ times, it at least begins to explain finite-Reynolds-number phenomena such as the generation of slugs of vorticity observed by experimentalists such as Wygnanski \& Champagne (1973). Of course in most situations there will be a number of competing mechanisms and transition to turbulence via the algebraic growth phenomenon (Schmid \& Henningson 1994) is also a possibility.

The input of larger disturbances than those proposed here would lead to nonlinear effects becoming important at earlier times in the evolution process. In such cases it is likely that the fully developed HPF state will never be attained and so-called bypass transition would occur. Our claim is that at large Reynolds number the critical amplitude suggested here marks the stability boundary between bypass transition and the decay of linear perturbations.

The author extends his thanks to Professor F. T. Smith for originally suggesting the nonlinear stability of Hagen-Poiseuille flow on the $O(R)$ length and time scales, and for many fruitful discussions over the years. The referees are also thanked for their constructive comments.

\section{REFERENCES}

Barnes, D. R. \& Kerswell, R. R. 2000 New results in rotating Hagen-Poiseuille flow. J. Fluid Mech. 417, 103-126.

Batchelor, G. K. 1967 An Introduction to Fluid Dynamics. Cambridge University Press.

Benney, D. J. \& Bergeron, R. F. 1969 A new class of nonlinear waves in parallel flows. Stud. Appl. Maths 48, 181-204.

Brown, S. N. \& Stewartson, K. 1978 The evolution of the critical layer of a Rossby wave. Part II. Geophys. Astrophys. Fluid Dyn. 10, 1-24. 
Davey, A. \& Drazin, P. G. 1969 The stability of Poiseuille flow in a pipe. J. Fluid Mech. 36, 209-218.

Draad, A. A., Kuiken, G. D. C. \& Nieuwstadt, F. T. M. 1998 Laminar-turbulent transition in pipe flow for Newtonian and non-Newtonian fluids. J. Fluid Mech. 377, 267-312.

GarG, V. K. \& Rouleau, W. T. 1972 Linear spatial stability of pipe Poiseuille flow. J. Fluid Mech. 54, 113-127.

Gill, A. E. 1965 On the behaviour of small disturbances to Poiseuille flow in a circular pipe. J. Fluid Mech. 21, 145-172.

GiLl, A. E. 1973 The least-damped disturbance to Poiseuille flow in a circular pipe. J. Fluid Mech. 61, 97-107.

Haberman, R. 1972 Critical layers in parallel flows. Stud. Appl. Maths 51, 139-161.

HABERMAN, R. 1976 Nonlinear perturbations of the Orr-Sommerfeld equation - asymptotic expansion of the logarithmic phase shift across the critical layer. SIAM J. Math. Anal. 7, 70-81.

Hall, P. \& Smith, F. T. 1991 On strongly nonlinear vortex/wave interactions in boundary-layer transition. J. Fluid Mech. 227, 641-666.

Han, G., Tumin, A. \& Wygnanski, I. 2000 Laminar-turbulent transition in Poiseuille pipe flow subjected to periodic perturbation emanating from the wall. Part 2. Late stage of transition. J. Fluid Mech. 419, 1-27.

Herbert, T. 1997 Parabolized stability equations. Annu. Rev. Fluid Mech. 29, 245-283.

Lin, C. C. 1955 The Theory of Hydrodynamic Stability. Cambridge University Press.

O'Sullivan, P. L. \& Breuer, K. S. 1994 Transient growth in circular pipe flow. II. Nonlinear development. Phys. Fluids 6, 3652-3664.

ReID, W. H. 1965 The stability of parallel flows. In Basic Developments in Fluid Dynamics, Vol. 1 (ed. M. Holt). Academic Press.

REYNOLDS, O. 1883 An experimental investigation of the circumstances which determine whether the motion of water will be direct or sinuous, and of the law of resistance in parallel channels. Phil. Trans. R. Soc. Lond. A 174, 935-982.

Salwen, H. \& Grosch, C. E. 1972 The stability of Poiseuille flow in a pipe of circular cross-section. J. Fluid Mech. 54, 93-112.

Schmid, P. J. \& Henningson, D. S. 1994 Optimal energy density growth in Hagen-Poiseuille flow. J. Fluid Mech. 277, 197-225.

SeXL, T. 1927 Zur stabilitatsfrage der Poiseuilleschen und Couetteschen stromung. Ann. Phys., Lpz. 83, 835-848.

Smith, F. T. \& Bodonyi, R. J. 1980 On the stability of the developing flow in a channel or circular pipe. Q. J. Mech. Appl. Maths 33, 293-320.

Smith, F. T. \& BodonyI, R. J. $1982 a$ Amplitude-dependent neutral modes in the Hagen-Poiseuille flow through a circular pipe. Proc. R. Soc. Lond. A 384, 463-489 (referred to herein as SB).

Smith, F. T. \& Bodonyi, R. J. $1982 b$ Nonlinear critical layers and their development in streamingflow stability. J. Fluid Mech. 118, 165-185.

StUART, J. T. 1963 Hydrodynamic stability. In Laminar Boundary Layers (ed. L. Rosenhead). Dover.

SZYMANSKI, P. 1932 Quelques solutions exactes des equations de l'hydrodynamique du fluide visqeux dans le cas d'un tube cylindrique. J. Math. Pure Appl. Series 9, 11, 67-107.

Toplosky, N. \& Akylas, T. R. 1988 Nonlinear spiral waves in rotating pipe flow. J. Fluid Mech. 190, 39-54.

Walton, A. G. 2001 The existence of neutral Rayleigh waves in the Hagen-Poiseuille flow through a pipe of circular cross-section. Stud. Appl. Maths 106, 315-335.

Wygnanski, I. J. \& Champagne, F. H. 1973 On transition in a pipe. Part 1. The origin of puffs and slugs and the flow in a turbulent slug. J. Fluid Mech. 59, 281-335. 